Mathematical Medicine and Biology (2011) 28, 357-384

doi:10.1093/imammb/dqr004

Advance Access publication on April 6, 2011

\title{
The influence of toxicity constraints in models of chemotherapeutic protocol escalation
}

\author{
ELEANOR A. J. BOSTON \\ School of Mathematics, University of Birmingham, Edgbaston, \\ Birmingham B15 2TT, UK \\ AND \\ EAMONN A. GAFFNEY* \\ Centre for Mathematical Biology, Mathematical Institute, \\ University of Oxford, Oxford OX1 3LB, UK \\ *Corresponding author: gaffney@maths.ox.ac.uk
}

[Received on 12 May 2010; revised on 16 February 2011; accepted on 22 February 2011]

\begin{abstract}
The prospect of exploiting mathematical and computational models to gain insight into the influence of scheduling on cancer chemotherapeutic effectiveness is increasingly being considered. However, the question of whether such models are robust to the inclusion of additional tumour biology is relatively unexplored. In this paper, we consider a common strategy for improving protocol scheduling that has foundations in mathematical modelling, namely the concept of dose densification, whereby rest phases between drug administrations are reduced. To maintain a manageable scope in our studies, we focus on a single cell cycle phase-specific agent with uncomplicated pharmacokinetics, as motivated by 5-Fluorouracil-based adjuvant treatments of liver micrometastases. In particular, we explore predictions of the effectiveness of dose densification and other escalations of the protocol scheduling when the influence of toxicity constraints, cell cycle phase specificity and the evolution of drug resistance are all represented within the modelling. For our specific focus, we observe that the cell cycle and toxicity should not simply be neglected in modelling studies. Our explorations also reveal the prediction that dose densification is often, but not universally, effective. Furthermore, adjustments in the duration of drug administrations are predicted to be important, especially when dose densification in isolation does not yield improvements in protocol outcomes.
\end{abstract}

Keywords: chemotherapeutic protocol modelling; age-structured modelling; Cell cycle phase specificity; toxicity constraints; drug resistance.

\section{Introduction}

Alterations in chemotherapy scheduling have frequently been considered with the objective of enhancing outcomes (Browder et al., 2000; Citron et al., 2003; Fallik et al., 2003), and a number of studies have been designed with the insight of mathematical modelling. A highly influential example is the hypothesis of Goldie and Coldman that minimizing drug resistance should be the primary objective, given an implicit assertion that resistance is the dominant reason for chemotherapy failure (Goldie and Coldman, 1979, 1983a,b, 1986, 1998; Goldie et al., 1982). Initial modelling studies revealed that this would require drug alternation [ibid], though clinical trials have typically failed to support the postulate that alternating treatments are superior to sequential protocols, as illustrated by De Placido et al. (1995), Sieber et al.

(C) The Author 2011. Published by Oxford University Press on behalf of the Institute of Mathematics and its Applications. All rights reserved. 
(2002) and Siodlak et al. (1990). Moreover, complications arising from cell cycle phase specificity and pharmacokinetics in modelling studies indicate that Goldie and Coldman's alternation hypothesis often need not hold (Gaffney, 2005). This highlights that scheduling concepts emerging from mathematical models should be explored to assess whether additional tumour biology places potential limitations on the conclusions that can be drawn.

Further seminal ideas in chemotherapy scheduling are dose intensification and densification. Respectively, these escalate the therapeutic protocol by increasing the dosage and reducing the time intervals between doses. Dose intensification, to the level of the maximally tolerated dose, is commonplace in chemotherapeutic scheduling and founded in the studies of Skipper (1965), though its effectiveness is occasionally questioned (Hahnfeldt et al., 2003; Haines, 2008; Markman, 1993). The concept of dose densification has emerged from the mathematical and computational modelling studies of Norton (1998b,a, 2001), based on earlier observations of tumour kinematics (Norton and Simon, 1977, 1986). Moreover, dose densification has been supported in a number of recent clinical trials (Citron et al., 2002, 2003), though in general it can yield equivocal results (Piccart et al., 2000). More recently, the consideration of more general protocol escalation have been considered by simultaneously altering the intensity and duration of drug administrations, with positive results in Phase-I human trials assessing safety regimes (Traina et al., 2008) and also in mouse models (Kolinsky et al., 2009).

Note that the modelling framework developed by Norton $(2001,2005)$ is typically based on Gompertzian tumour growth with alterations in the rest phase between doses. Factors such as vascularity, oxygen partial pressures, acidity, excess glycolysis, toxicity and drug resistance illustrate features of the tumour microenvironment that are not explicitly considered in the modelling. Arguably, the influence of all but the last two, these features are captured implicitly by considering Gompertzian tumour kinetics and Norton and Simon's hypothesis that the tumour regression rate with treatment is proportional to the unperturbed growth rate. However, the latter two, toxicity and drug resistance, are critical reasons for chemotherapeutic failure and thus merit further attention.

It has been postulated that dose densification in particular need not exacerbate toxicity. The reasoning is that normal tissues may be more perturbed by protocol duration than by reducing the interdosing time due to the fact that they are much closer to their saturation levels (Norton and Simon, 1977; Norton, 2008); this could emerge from the lower growth fraction of normal tissue for example. Empirically, however, one can find examples where dose densification exacerbates toxicity (Piccart et al., 2000) and, conversely, where it does not (Citron et al., 2003; Simon and Norton, 2006). These equivocal observations nonetheless highlight the need to consider toxicity in models of protocol scheduling to assess when toxicity may impinge on protocol outcomes and when Norton's framework requires the inclusion of further tumour biology.

While there have been numerous studies considering toxicity in explorations of resonances with timings based on cell cycle durations guiding protocol schedules (e.g. Agur et al., 1988, 1992; Cojocaru and Agur, 1992; Dibrov, 1998; Ubezio et al., 1994; Webb, 1990), modelling investigations focussing on resistance and/or the influence of extensive protocol escalation typically do not consider this important constraint in detail. In particular, there are no studies accommodating both toxicity and Goldie and Coldman's carefully motivated framework for modelling the random evolution of resistance, based upon the application of Luria and Delbrück's Nobel Prize winning concepts to cancer cell systems (Jaffrezou et al., 1994; Law, 1952; Summers and Handshumacher, 1973). This is most likely due to the fact that Goldie and Coldman focussed on studying the effects of adjusting the alternation of drugs with identical sensitive cell kill rates, whereby one is automatically comparing protocols of equal toxicity. Consequently, simulations of the influence of drug toxicity when the evolution to resistance is present are underdeveloped. 
In addition, the Norton framework (Norton, 2001, 2005) does not explicitly consider the interplay between protocol timings and the passage of cells through the cell cycle, despite the fact numerous therapeutic interventions rely on cell cycle phase specificity to sufficiently differentiate between tumour and host. Furthermore, given protocol timings explicitly influence the distribution of cells through the different phases of the proliferative cycle, the extent to which cell cycle sanctuaries can reduce treatment effectiveness is not immediately apparent (e.g. Gaffney, 2004). Consequently, one cannot immediately infer the effectiveness of protocol escalation for cell cycle phase-specific treatments from modelling founded upon phase-independent cell kill mechanisms.

Our objective is therefore to conduct a modelling exploration of whether protocol escalations, or indeed other scheduling adjustments, can yield predictions of therapeutic improvements given the explicit consideration of the cell cycle, drug toxicity and drug-resistance evolution. Assuming linear cell kill, which is frequently but not universally observed (Frei and Antman, 2000), we will consider maximally tolerated dosages and then subsequently explore further protocol alterations by dose densification and also by increases in the dose administration duration. In particular, our studies will focus on a specific context to narrow the scope of the investigation; we will address adjuvant protocols in the colorectal setting aimed at eliminating residual liver neoplastic deposits following surgical resection of the primary tumour. These are of fundamental concern due to the incessant tendency of colorectal micrometastases to impregnate and establish within the liver via the hepatic portal vein; the consequent secondary disease has an extremely poor prognosis (Adam, 2006; Midgley and Kerr, 1999).

The principle agents for such adjuvant treatments are based upon 5-Fluorouracil (5-FU), a drug with a very low plasma half life and a dominant mode of action in S-phase, that is the DNA-synthesis stage of the cell cycle as detailed in Fig. 1 (Midgley and Kerr, 2000; Nicum et al., 2000). In particular, a derivative, 5-Fluoro-2' deoxyuridine (FUDR), is often continuously administered via a hepatic arterial pump or infusion (Kemeny et al., 1999; Kemeny and Fata, 2001; Tebbutt et al., 2002) and predominantly absorbed by the liver (Ensminger et al., 1978). The dose limiting toxicity for this intervention is hepatobiliary, with reports of inflammation surrounding the biliary tree together with fibrosis of the bile ducts and secondary chemical hepatitis (Cohen and Kemeny, 2003; Skitzki and Chang, 2002), indicating that the biliary duct epithelia are the most susceptible tissues. This focus on the regional delivery of

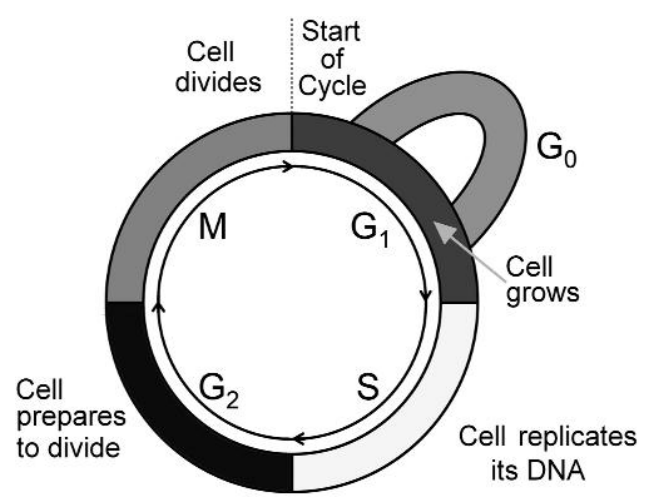

FIG. 1. A schematic of the cell cycle model. A cell undergoing proliferation moves through a number of stages. The first is $G_{1}$, with extensive cytoplasmic growth, followed by S-phase when the DNA is replicated, the $G_{2}$ rest phase and then M-phase, where mitosis proceeds ending in cell division. Cells can also transfer back and forth between the $\mathrm{G}_{1}$-phase and a non-cycling compartment, $\mathrm{G}_{0}$, where they are quiescent in terms of proliferation events. 
a readily absorbed, but unstable, drug together with the relative absence of systemic toxicity allows the neglect of detailed drug pharmacokinetics.

Thus, we will consider a model for the scheduling of a single S-phase-specific agent delivered via intermittently continuous regional infusions, taking into account the evolution to drug resistance using the modelling framework of Goldie and Coldman. Toxicity will be explicitly considered, though the presence of cell cycle phase specificity entails that feasible forms of maximally tolerated chemotherapeutic dosages or accumulation levels cannot be readily postulated, in contrast with many previous modelling explorations of toxicity and scheduling (e.g. Afenya, 2001; Barbolosi and Iliadis, 2001; Costa et al., 1995; Matveev and Savkin, 2005; Panetta and Adam, 1995). Hence, the inclusion of toxicity requires the explicit consideration of the dose-limiting healthy cell dynamics. These models are constructed in Section 2 and explored in Section 3, with conclusions drawn in Section 4, focussing on the effectiveness of dose densification and other scheduling alterations.

\section{Model development}

\subsection{Healthy cell population model}

To consider the influence of an S-phase-specific chemotherapeutic on susceptible liver tissue insulted with regional chemotherapy, we require a model for the response and regrowth of tissue, accounting for the fact that drug effect is contingent upon the cell cycle phase, which in turn is dependent on the history of the protocol.

Firstly, the healthy cell model should track populations as they move through the cell cycle, imposing an age structure on the modelling. A fundamental framework for this in terms of hyperbolic partial differential equations has already been developed (Chiorino et al., 2001; Gaffney, 2004; Webb, 1990). Furthermore, it has been explicitly validated for the progression of tumour cells through the cell cycle by comparing predictions and observations for oscillations in labelling indices following the release from a cell cycle arrest (Chiorino et al., 2001).

For healthy tissue, this modelling framework must be supplemented with growth regulation preventing excess cell proliferation; however, the biochemical mechanisms for such control with hepatocytes and biliary duct epithelial cells, cholangiocytes, are poorly understood (Court et al., 2002; Marzioni et al., 2005). Consequently, we consider a simple volume control, which is consistent with general observations that liver size regulation is exquisitely controlled by volume (Court et al., 2002) and liver regeneration is accompanied by regulated cholangiocyte proliferation (LeSage et al., 1996). Thus, once the susceptible population reaches a critical volume, it is assumed that cells pass from the $\mathrm{G}_{1}$ cell cycle phase into the $G_{0}$ rest phase. Conversely, below the critical population volume, cells are recruited into cycle from $\mathrm{G}_{0}$; the modelling assumes a ten-hour delay from signal to recruitment given general observations of cultured mammalian cells as they leave $\mathrm{G}_{0}$ to enter the cell cycle (Wolfe, 1995; Zetterberg et al., 1995).

We note that for a wide range of mammalian cell types, cell growth is observed to be linear in time (Conlon and Raff, 2003) and thus, given the cell cycle parameters used below, with the $\mathrm{G}_{1}$ phase duration approximately the cell cycle time, cell growth occurs predominantly in the $\mathrm{G}_{1}$-phase. Hence, as a modelling simplification, cell volume increases are only allowed within the $\mathrm{G}_{1}$-phase; furthermore, it is also assumed that cells grow to twice their volume during the cell cycle and divide symmetrically. These considerations lead us to the following framework for modelling the dynamics of the dose-limiting tissue:

1. Movement from the $\mathrm{G}_{1}$-phase to the $\mathrm{G}_{0}$-phase occurs if the population volume is above a critical level, in which case cell volume growth in $\mathrm{G}_{1}$ is halted. The converse also holds if the population 
volume is below a critical value, inducing cell volume growth in $\mathrm{G}_{1}$ and the passage of cells from $\mathrm{G}_{0}$ to $\mathrm{G}_{1}$, though the latter suffers a ten-hour delay between signal and effect.

2. All cytoplasmic growth for a cell in cycle occurs during $\mathrm{G}_{1}$ at a constant rate when cell growth is allowed by the volume regulation. As a result of this approximation, a cell must reach double its initial size on leaving $\mathrm{G}_{1}$ after which the cell is taken to not grow any further during the cycle.

3. Movement between subsequent phases of the cell cycle occurs after a specific, fixed, duration in each phase, consistent with general observations of the cell cycle (Abdreeff et al., 2000).

4. While cell division occurs towards the end of the M-phase, we approximate this as an instantaneous process at the very end of M-phase. We assume symmetrical division only.

5. Small amounts of cell apoptosis occur at a constant rate in the quiescent, $\mathrm{G}_{0}$, population.

We also have to consider the effects of the chemotherapeutic on healthy tissue. In particular, 5-FU is known to cause DNA double strand breaks during $\mathrm{S}$-phase, resulting in $\mathrm{G}_{2}$-phase arrest (Okamoto et al., 1996). Thus, the chemotherapeutic is modelled as inducing movement of S-phase cells into a damaged cycling compartment; once these damaged cells reach the $\mathrm{G}_{2}$ cell cycle checkpoint, they enter an apoptotic compartment, leading to eventual cell death.

The modelling of these concepts therefore requires the following compartments:

- $G_{1}(v, t)$ and $G_{0}(v, t)$, which are the densities for cells of volume $v$ and at time $t$ in the $\mathrm{G}_{1-}$ and $\mathrm{G}_{0}$-phases, respectively.

- $S(a, t)$, the age density of viable cells in the S-phase at age $a$ and time $t$, where age is the duration of a cell in the compartment.

- $G_{\mathrm{m}}(a, t)$ : the age density of cells in a combined $\mathrm{G}_{2}$ and M-phase at age $a$ and time $t$.

- $S_{\mathrm{d}}(a, t)$, the age density of cells in the S-phase damaged by chemotherapy at age $a$ and time $t$.

- $A(t)$, the number of apoptotic cells due to chemotherapy at time $t$.

We also denote the total cell population volume by $V(t)$; in the following, we capture these ideas in a modelling framework, which is summarized in Fig. 2.

2.1.1 A model of the $S, G_{2}$ - and M-phases. Balance equations for cells in the S-phase and the $\mathrm{G}_{2} / \mathrm{M}$ phases, with an S-phase chemotherapeutic inducing a first-order cell kill, are given by

$$
\begin{aligned}
\frac{\partial S}{\partial t}+\frac{\partial S}{\partial a} & =-\Phi S, \quad a \in\left[0, a_{2}\right], \\
\frac{\partial G_{\mathrm{m}}}{\partial t}+\frac{\partial G_{\mathrm{m}}}{\partial a} & =0, \quad a \in\left[0, a_{3}\right] .
\end{aligned}
$$

Here, $\Phi$ denotes the rate S-phase chemotherapy irreparably damages healthy cells and $a_{2}, a_{3}$ denote the transit time for cells on their passage through S-phase and the $\mathrm{G}_{2} / \mathrm{M}$ phases. These compartmental transit times are observed to be effectively invariant in both healthy and tumour tissues (Abdreeff et al., 2000).

2.1.2 Cell loss due to chemotherapy. Chemotherapy damaged cells, en-route to cell death, enter the damaged compartment where they continue to cycle. On reaching the $\mathrm{G}_{2}$ checkpoint, they enter the 


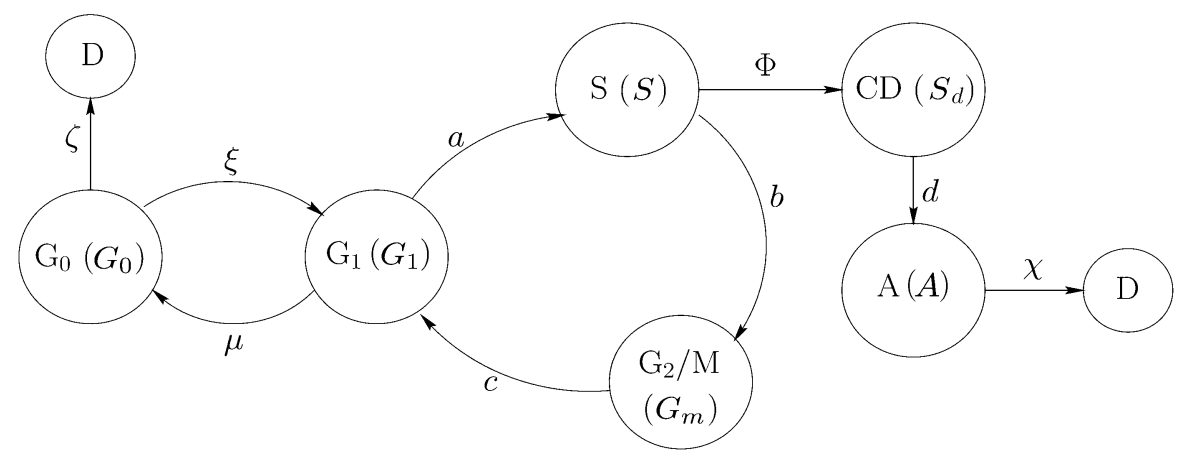

FIG. 2. A schematic diagram of the healthy cell population model, with cell transfer to $\mathrm{G}_{0}$ occurring at rate $\mu$, to $\mathrm{G}_{1}$ at rate $\xi$, and quiescent cells apoptosing at rate $\zeta$ into a dead cell compartment (D). Progression through the cell cycle is marked at the end of each phase by $a, b$ and $c$, with $d$ representing the passage of chemotherapy damaged cells (CD) into the apoptotic compartment (A). The rate healthy cells are irreparably damaged is denoted by $\Phi$, while the parameter $\chi$ represents the rate of death of these damaged cells once they enter the apoptotic compartment. The model variable associated with each compartment is given in brackets.

apoptotic compartment leading to eventual cell death at rate $\chi$. In terms of the damaged cell density, $S_{\mathrm{d}}$, and the number of apoptotic cells, $A$, these processes are represented by the equations

$$
\begin{aligned}
\frac{\partial S_{\mathrm{d}}}{\partial t}+\frac{\partial S_{\mathrm{d}}}{\partial a} & =\Phi S(a, t), \quad a \in\left[0, a_{2}\right], \\
\frac{\mathrm{d} A}{\mathrm{~d} t} & =S_{\mathrm{d}}\left(a_{2}, t\right)-\chi A .
\end{aligned}
$$

2.1.3 A model of the $G_{0}$-phase. To incorporate the change in the rate of transfer between the $\mathrm{G}_{1}$ cell cycle phase and the $\mathrm{G}_{0}$ cell cycle phase, which depends on the cell population volume $V(t)$, we define a step function $H_{0}$ by

$$
H_{0}\left[V(t)-V_{\max }\right]= \begin{cases}0 & \text { if } V(t) \geqslant V_{\max } \\ 1 & \text { if } V(t)<V_{\max }\end{cases}
$$

where $V_{\max }$ is the critical volume associated with the volume regulation mechanism. We also denote the time delay between signal and recruitment from $G_{0}$ by $\Delta$, the rate at which $\mathrm{G}_{0}$ cells are lost by $\zeta$ and transfer coefficients to and from the $\mathrm{G}_{0}$ compartment, respectively, by $\mu$ and $\xi$. Then the balance equation for cells in $\mathrm{G}_{0}$ is

$$
\frac{\partial G_{0}}{\partial t}=\mu\left(1-H_{0}\left[V(t)-V_{\max }\right]\right) G_{1}(v, t)-\xi\left(H_{0}\left[V(t-\Delta)-V_{\max }\right]\right) G_{0}(v, t-\Delta)-\zeta G_{0}(v, t),
$$

with $v \in\left[v_{*}, 2 v_{*}\right]$, where $v_{*}$ denotes the cell volume immediately following mitosis. Note that the density $G_{0}$ implicitly tracks cell volumes so that volume is conserved should $\mathrm{G}_{0}$ cells begin cycling by re-entering the $G_{1}$ cell cycle phase. Finally, the loss of $G_{0}$ cells in the absence of chemotherapy is very small compared to total cell numbers, so we do not track healthy cells which are committed to apoptosis via this route. 
2.1.4 A model of the $G_{1}$-phase. We first of all consider the case where the total population volume $V(t)$ is such that $V(t)<V_{\max }$, so that cells are being actively recruited into the cycling $\mathrm{G}_{1}$ phase from $\mathrm{G}_{0}$. Then the cell balance for the $\mathrm{G}_{1}$-phase yields

$$
\frac{\partial G_{1}}{\partial t}+\dot{v} \frac{\partial G_{1}}{\partial v}=\xi G_{0}(v, t-\Delta), \quad V(t)<V_{\max }
$$

for $v \in\left[v_{*}, 2 v_{*}\right]$, with $\dot{v}$ representing the rate of cell volume change with duration in the $\mathrm{G}_{1}$ compartment. The movement of cells into the $\mathrm{G}_{1}$-phase from the mitotic (M)-phase of the previous cycle is incorporated via the boundary conditions when cells are cycling (see Section 2.1.6 below for further details). Note the assumption that all cytoplasmic growth occurs in $\mathrm{G}_{1}$ entails that the maximum cell volume is $2 v_{*}$, giving the range of $v$. Under maximal growth conditions, the cytoplasmic growth rate is taken to be constant and, with $a_{*}^{s}$ denoting the transit time of a cell through $\mathrm{G}_{1}$ under these conditions, we have

$$
\dot{v}=v_{*} / a_{*}^{s}, \quad V(t)<V_{\max } .
$$

In contrast, growth is arrested if $V(t) \geqslant V_{\max }$. Thus, in general, we have

$$
\dot{v}(t)=\frac{v_{*}}{a_{*}^{s}} H_{0}\left[V(t)-V_{\max }\right]
$$

When $\dot{v}=0$, cells are arrested in the $\mathrm{G}_{1}$ compartment and slowly transfer into $\mathrm{G}_{0}$. Cells are still taken to leave the $G_{2} / M$ compartment following mitosis. They then enter $G_{1}$, at which point they no longer cycle. Hence, for $v \in\left[v_{*}, 2 v_{*}\right]$ we have the balance equation for $G_{1}(v, t)$ is given by

$$
\frac{\partial G_{1}}{\partial t}=-\mu G_{1}(v, t)+2 \delta\left(v-v_{*}\right) G_{\mathrm{m}}\left(a_{2}, t\right), \quad V(t) \geqslant V_{\max }
$$

Note that the term $2 \delta\left(v-v_{*}\right) G_{\mathrm{m}}\left(a_{2}, t\right)$ accounts for cells entering $\mathrm{G}_{1}$ following mitotic cell division.

In summary, we have that the governing conservation equation for cells in the $G_{1}$ compartment is given by

$$
\begin{aligned}
\frac{\partial G_{1}}{\partial t}+\dot{v} \frac{\partial G_{1}}{\partial v}= & \xi\left(H_{0}\left[V(t-\Delta)-V_{\max }\right]\right) G_{0}(v, t-\Delta)-\mu\left(1-H_{0}\left[V(t)-V_{\max }\right]\right) G_{1}(v, t) \\
& +2\left(1-H_{0}\left[V(t)-V_{\max }\right]\right) \delta\left(v-v_{*}\right) G_{\mathrm{m}}\left(a_{2}, t\right)
\end{aligned}
$$

for $v \in\left[v_{*}, 2 v_{*}\right]$, with $\dot{v}$ given by (6). For the numerical solutions below a regularized approximation for the Dirac-delta function is used, whereby

$$
\delta\left(v-v_{*}\right) \approx\left\{\begin{array}{l}
\frac{1}{\epsilon_{*}} v \in\left[v_{*}, v_{*}+\epsilon_{*}\right] \\
0 \text { otherwise }
\end{array}\right.
$$

with $\epsilon_{*} \ll v_{*}$ given by the numerical grid spacing in a finite-difference scheme. 
2.1.5 The healthy cell population volume. Note that, with the assumptions of cell growth solely in the $G_{1}$ cell cycle phase and cell splitting at the very end of the $M$ cell cycle phase, cells in the $G_{2}$ and $\mathrm{M}$ compartments have volume $2 v_{*}$. Hence, the total healthy cell population volume is given in terms of the compartment cell population densities by

$$
\begin{aligned}
& V(t)=\int_{v_{*}}^{2 v_{*}} v\left[G_{1}(v, t)+G_{0}(v, t)\right] \mathrm{d} v+2 v_{*} A(t)+2 v_{*}\left[\int_{0}^{a_{2}}\left\{S(a, t)+S_{\mathrm{d}}(a, t)\right\} \mathrm{d} a\right. \\
& \left.+\int_{0}^{a_{3}} G_{\mathrm{m}}(a, t) \mathrm{d} a\right] .
\end{aligned}
$$

2.1.6 Boundary and initial conditions. The boundary conditions for the hyperbolic equations arise from conservation principles for fluxes between the compartments. Thus, we have

$$
G_{\mathrm{m}}(0, t)=S\left(a_{2}, t\right), \quad S(0, t)=G_{1}\left(2 v_{*}, t\right) \dot{v}(t), \quad S_{\mathrm{d}}(0, t)=0 .
$$

In addition, when the total population volume $V(t)$ is such that $V(t)<V_{\max }$, so that $\dot{v}>0$, we have

$$
\dot{v}(t) G_{1}\left(v_{*}, t\right)=2 G_{\mathrm{m}}\left(a_{3}, t\right) .
$$

In contrast, when the total volume exceeds $V_{\max }$ and the cell cycle arrests we have $\dot{v}(t)=0$. Thus, at this point, equation (8), which governs the evolution of $G_{1}(v, t)$, is degenerate and the boundary condition (10) is redundant.

Due to the time delay, the initial conditions are specified for the temporal interval $t \in \mathcal{T}=[-\Delta, 0]$. Initially, it is assumed that there are no damaged or apoptotic cells so that we have $A=S_{\mathrm{d}}=0$ for $t \in \mathcal{T}$. The remaining initial conditions are of the form

$$
\begin{aligned}
G_{0}(v, t) & =G_{0}^{0}(v), \quad G_{1}(v, t)=G_{1}^{0}(v), \\
S(a, t) & =S^{0}(a), \quad G_{\mathrm{m}}(a, t)=G_{\mathrm{m}}^{0}(a),
\end{aligned}
$$

where $t \in \mathcal{T}$. The functions $G_{0}^{0}(v), G_{1}^{0}(v), S^{0}(a)$ and $G_{\mathrm{m}}^{0}(a)$ are taken to be those arising from the large time asymptote of numerical simulations in the absence of chemotherapy.

\subsection{Tumour cell population model}

We need to consider the influence of an S-phase-specific chemotherapeutic on tumour tissue, taking into account the evolution to drug resistance using Goldie and Coldman's modelling framework. Given there is no growth saturation mechanism in the tumour model below and the motivating context is adjuvant chemotherapy, the modelling will be exploring the influence of treatment on an exponentially growing micrometastasis, as may be apparent for a freshly vascularized nodule.

We require an age-structured model tracking tumour cells through the cell cycle phases and the influence of the treatment in S-phase, together with a small mutation rate that governs the probability that a tumour cell will become resistant. As Goldie and Coldman (1979, 1983a,b, 1986, 1998) have carefully documented and motivated, this mutation rate can be modelled as being independent of the details of the drug protocol; resistance is driven by random mutation rather than by a Lamarckian response to the 
chemotherapeutic. Furthermore, since the mutation to resistance is a rare event, at least for an individual cell, and initially no resistant cells are assumed to be present, stochastic modelling is required. However, with the assumption that mutation to resistance is absolute, ultimately leading to ineffective treatment, we simply need to track the probability that there are no cells that have become resistant, simplifying the modelling framework.

Furthermore, while many mutations can occur due to polymerase errors during DNA replication, they can also be due to chromosomal instabilities though only one mechanism is typically dominant in colorectal tumours (Loeb et al., 2003). Here, for model simplification, we take it that the resistance conferring mutations only occur in the DNA synthesis, S-phase; we also assume that the mutation rate is constant throughout this phase of the cell cycle.

Finally, note that the initial tumour volume is negligible with respect to the volume constraint of the healthy cells. Given we simply require the probability that resistance initiates, the model is only required to be valid up to the point that resistance occurs. Furthermore, in the current framework, a chemotherapeutic protocol cannot possibly be successful unless it eliminates the sensitive tumour cells within the nodule before resistance sets in. Thus, unless protocol failure is certain, the nodule is always shrinking until resistance initially occurs. Hence, in the required domain of validity for the modelling, the tumour nodule is always shrinking and hence always has effectively no influence on the volume constraint of the healthy cells. This very important simplification allows a substantial decoupling between the dynamics of the healthy and tumour cell models.

2.2.1 Tumour cell model compartments. The model of tumour cells in cycle under the influence of an S-phase chemotherapeutic is developed below, with lower case letters reserved for tumour densities:

- $s(a, t)$, the age density of tumour cells in S-phase at age $a$ and time $t$, with $a \in\left[0, a_{2}\right)$.

- $g_{\mathrm{m}}(a, t)$, the age density of tumour cells in a combined $\mathrm{G}_{2}$ and M-phase at age $a$ and time $t$, with $a \in\left[0, a_{3}\right)$.

- $g_{1}(a, t)$, the age density of tumour cells in the $\mathrm{G}_{1}$-phase of the cell cycle at age $a$ and time $t$. In this compartment $a \in\left[0, a_{1}\right)$, where $a_{1}$ is the maximum duration of the transit time. However, in contrast to the duration of the S-phase $\left(a_{2}\right)$ and the $\mathrm{G}_{2} / \mathrm{M}$ phase $\left(a_{3}\right)$, the transit time of the $\mathrm{G}_{1}$-phase is variable (Abdreeff et al., 2000; Cooper, 2000). This is especially true for cancer cells, which is postulated to arise from the loss of normal growth control being linked to the proto-oncogene behaviour of cyclins of the $\mathrm{G}_{1}$-phase (Musgrove et al., 1994). Thus, the tumour cell $\mathrm{G}_{1}$ transit time is represented by a random variable below.

Note that tumour cells are assumed to be uninfluenced by inhibitory controls and to be undergoing rapid self-similar exponential growth and hence a quiescent $\mathrm{G}_{0}$ compartment is not considered. In particular, while quiescence does occur in tumours, and would progressively slow down growth rates as cells come out of cycle, we are considering freshly vascularized micrometastases in the context of adjuvant therapy. The sensitive cell numbers are relatively small and assumed to be insufficient for the decelerations observed beyond the early stages of the non-exponential Gompertz or Gomp-exp fits to tumour growth data (e.g. Wheldon, 1988).

Finally, observe that apoptotic tumour cells are not tracked in that once a tumour cell is committed to apoptosis, it is assumed to die and, thus within the current framework, no longer influences the dynamics. 
2.2.2 A model of the $S, G_{2}$ - and M-phases. The cell density within the S-phase and the $\mathrm{G}_{2} / \mathrm{M}$ phase are governed by the following balance equations:

$$
\begin{aligned}
\frac{\partial s}{\partial t}+\frac{\partial s}{\partial a} & =-[\Phi+\alpha] s, \quad a \in\left[0, a_{2}\right], \\
\frac{\partial g_{\mathrm{m}}}{\partial t}+\frac{\partial g_{\mathrm{m}}}{\partial a} & =0, \quad a \in\left[0, a_{3}\right],
\end{aligned}
$$

where $\Phi$ represents the first-order cell kill rate of the chemotherapeutic and $\alpha \ll a_{2}^{-1}$ is the mutation rate.

2.2.3 A model of the $G_{1}$-phase. Balancing cells for the $\mathrm{G}_{1}$-phase gives

$$
\frac{\partial g_{1}}{\partial t}+\frac{\partial g_{1}}{\partial a}=-\gamma(a) g_{1}, \quad a \in\left[0, a_{1}\right]
$$

where the rate cells leave this phase, $\gamma(a)$, is dictated by the probability distribution function of the duration of $\mathrm{G}_{1}$, denoted $f(a)$. In particular, we have (Chiorino et al., 2001)

$$
\gamma(a)=\frac{f(a)}{1-\int_{0}^{a} f\left(a^{\prime}\right) \mathrm{d} a^{\prime}}
$$

The key feature of the distribution $f(a)$ is that it possesses a single peak centred at the median $\mathrm{G}_{1}$ transit time. Below we take it to be a truncated Gaussian

$$
f(a)=N_{f} \exp \left(-\frac{\left(a-a_{*}^{c}\right)^{2}}{2 \sigma^{2}}\right), \quad a \in\left[0, a_{1}\right],
$$

where $a_{*}^{c}$ is the median of the unimodal $\mathrm{G}_{1}$ duration and $N_{f}$ is a normalization constant that is fixed so as to ensure that

$$
\int_{0}^{a_{1}} f(a) \mathrm{d} a=1 .
$$

As the tumour cell cycle time differs from the $\mathrm{G}_{1}$ transit time by a constant, the variance of both these random variables is governed by $\sigma^{2}$.

2.2.4 Boundary and initial conditions. Cell balance in the fluxes between compartments yields the following boundary conditions:

$$
g_{1}(0, t)=2 g_{\mathrm{m}}\left(a_{3}, t\right), \quad s(0, t)=\int_{0}^{a_{1}} \gamma\left(a^{\prime}\right) g_{1}\left(a^{\prime}, t\right) \mathrm{d} a^{\prime}, \quad g_{\mathrm{m}}(0, t)=s\left(a_{2}, t\right) .
$$

The initial distribution of cells among the compartments is taken to be governed by the large time asymptotic distribution predicted by the model in the absence of chemotherapy. Using $N_{0}$ to denote the total initial tumour cell number, this asymptotic distribution is given at leading order in an expansion of $\alpha a_{2} \ll 1$ by (Gaffney, 2004)

$$
g_{1}(a, 0)=\frac{2 N_{0} \ln 2}{T_{\mathrm{c}}} \mathrm{e}^{-\lambda a} \mathrm{e}^{-\int_{0}^{a} \gamma(s) \mathrm{d} s}, \quad s(a, 0)=\frac{N_{0} \ln 2}{T_{\mathrm{c}}} \mathrm{e}^{\lambda\left(a_{2}+a_{3}\right)} \mathrm{e}^{-\lambda a}, \quad g_{\mathrm{m}}(a, 0)=s(a, 0) \mathrm{e}^{-\lambda a_{2}},
$$


with $\lambda \stackrel{\text { def }}{=}[\ln 2] / T_{\mathrm{c}}$ and $T_{\mathrm{c}}$ denoting the median tumour cell cycle time. The distribution can also be found numerically.

2.2.5 The probability of no resistance and the probability of success. Let $p_{0}(t)$ denote the probability that no resistant cells are present at time $t$; we assume that there are no resistant cells initially so that $p_{0}(0)=1$. The assumption that the emergence of resistance is equivalent to protocol failure entails that $p_{0}(\infty)$ is the probability of success; in particular, the elimination of the non-resistant tumour cells is necessary but not sufficient for $p_{0}(\infty)>0$. Given the resistance is generated randomly within S-phase at a constant rate $\alpha$ we have, for $\delta t$ sufficiently small,

$$
p_{0}(t+\delta t)=p_{0}(t)\left[1-\delta t \int_{0}^{a_{2}} \alpha s\left(a^{\prime}, t\right) \mathrm{d} a^{\prime}\right]+o(\delta t)
$$

Dividing by $\delta t$ and taking the limit $\delta t \rightarrow 0$, whereby the $o(\delta t)$ term also tends to zero, yields an ordinary differential equation for $p_{0}(t)$ with initial condition $p_{0}(0)=1$ and solution

$$
p_{0}(t)=\exp \left[-\alpha \int_{0}^{t} \int_{0}^{a_{2}} s\left(a^{\prime}, t^{\prime}\right) \mathrm{d} a^{\prime} \mathrm{d} t^{\prime}\right] .
$$

Note that while first-order cell kill kinetics entail that sensitive tumour cell elimination is not mathematically possible in the model for finite time, one may reasonably take very small cell numbers (below unity for example) as representing total sensitive cell kill. With this qualification, sensitive cell regrowth cannot occur once sensitive cells are eliminated and $p_{0}(\infty)$ is given to an excellent approximation by numerically simulating the model with the chemotherapeutic protocol active until $p_{0}(t)$ asymptotes at an appropriate level of tolerance. This is implicit below in the numerical simulations as is the modelling assumption that continuing therapy indefinitely, beyond the point of effective sensitive cell elimination, will not yield further benefits. We briefly remark that this is seen clinically in the motivating context of adjuvant therapies of colorectal cancer following resection of the primary tumour, where increasing trial treatment durations from a total of 6 months to 12 months has been concluded to have no effect on outcome (Midgley and Kerr, 1999, 2000).

\subsection{The chemotherapeutic protocol}

We consider the action of a single S-phase-specific drug on both healthy and tumour cells, assuming the drug is delivered and decays rapidly, as with infusions and intermittent infusions of 5-FU, commonly used in the context of adjuvant colorectal cancer therapies. Hence, in our model, the chemotherapeutic is represented by a square wave function of application, with immediate drug action once applied and no drug action as soon as the administration has ceased.

Thus, a protocol is considered such that each chemotherapy cycle consists of a drug application for a duration of $t_{1}$, followed by a rest phase of duration $k t_{1}$. This cycle is repeated to give a protocol represented by

$$
\begin{array}{lccccc}
\text { Drug status } & \text { on } & \text { off } & \text { on } & \text { off } & \ldots \\
\text { Duration } & t_{1} & k t_{1} & t_{1} & k t_{1} & \ldots
\end{array}
$$

We define $\phi(t)$ to be the indicator function for the presence of the drug, as depicted in Fig. 3. The cell kill rate due to the drug is taken to be

$$
\Phi \stackrel{\text { def }}{=} \hat{m} \phi(t) ;
$$




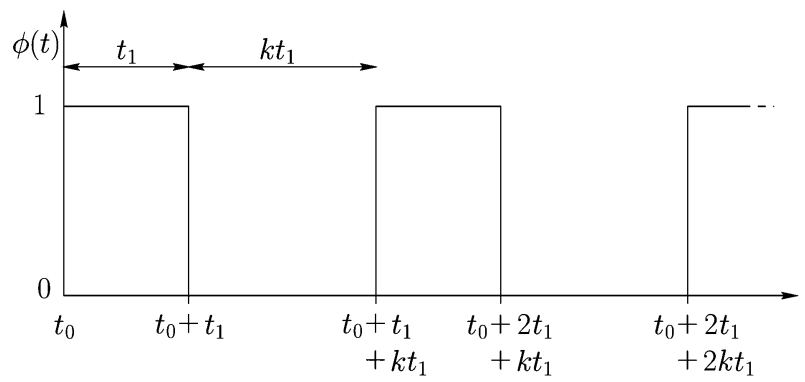

FIG. 3. A schematic diagram of the protocol where a repeated chemotherapeutic cycle consists of a drug application for duration $t_{1}$ followed by a rest phase for duration $k t_{1}$, with chemotherapy initiation depicted at time $t_{0}$. The function $\phi(t)$ represents the indicator function for the presence of the drug at time $t$.

thus increasing $\hat{m}$ corresponds to dose intensification. We implicitly assume that cell kill saturation does not occur so that any increase in cell kill intensity considered in the modelling can be realized; this is commonly reported in the form of linear cell kill, but it certainly is not universal (Frei and Antman, 2000).

It is also convenient below to define $\hat{\psi}$ to be the area under curve of drug cell kill for a single administration of the chemotherapeutic. Thus, for $\tau$ representing any fixed time after chemotherapy initiation, we have

$$
\hat{\psi} \stackrel{\text { def }}{=} \hat{m} \int_{\tau}^{\tau+k t_{1}+t_{1}} \phi\left(t^{\prime}\right) \mathrm{d} t^{\prime}=\hat{m} t_{1} ;
$$

note that $\hat{\psi}$ is dimensionless as $\hat{m}$ is a rate of cell kill.

In manipulating the protocol in the explorations below, we can attempt to escalate the protocol by reducing the rest phase (dose densification) or by lengthening the duration of the administration. In contrast, the dose intensity, $\hat{m}$, is dictated by the maximally tolerable dose given the toxicity constraint, which we now detail.

2.3.1 Toxicity constraint. The toxicity constraint, which limits the extent to which the chemotherapeutic protocol can be escalated, is expressed in terms of the total number of healthy cells in the $\mathrm{G}_{0}$-phase relative to its equilibrium value in the absence of chemotherapy. In particular, we require

$$
\frac{\int_{\nu_{*}}^{2 \nu_{*}} \mathrm{~d} \nu G_{0}(\nu, t)}{\int_{\nu_{*}}^{2 \nu_{*}} \mathrm{~d} \nu G_{0}^{\text {equilibrium }}(v)} \geqslant \Upsilon,
$$

with $\Upsilon \in(0,1)$. While it is difficult to estimate $\Upsilon$, we typically take $\Upsilon=0.05$ which entails that the toxicity constraint becomes limiting only once an exhaustion of the $\mathrm{G}_{0}$ reserve is imminent, but without a traumatic loss of healthy cell number. Below, by assuming the use of the maximally tolerated dosage, we consider the maximal cell kill of the chemotherapeutic,

$$
\hat{m}_{\max } \stackrel{\text { def }}{=} m,
$$

allowed by the toxicity constraint. 
For a given scheduling, finding the maximally tolerated cell kill intensity, $m$, or equivalently the maximally tolerated cell kill area under curve,

$$
\hat{\psi}_{\max } \stackrel{\text { def }}{=} \psi=m t_{1},
$$

is computationally intensive. It requires solving the healthy cell equations for different dosage intensities, and using a root finder to find the intensity where the bound in inequality (19) is just attained, to within tolerance levels. Once the maximal cell kill area under the curve, $\psi$, becomes sufficiently large, the cell kill per dose administration is such that essentially all cells in or entering S-phase are killed to high accuracy. Further intensity increases have minimal effects on the level of cell kill; utilizing this observation entails numerical savings may be made by capping the maximum intensity in the root finder (the cell kill area under curve, which is non-dimensional, is capped at twenty in the simulations due to these observations). Alternatively, this cap could be viewed as a sharp limit to drug effectiveness once the area under curve is sufficiently large with a resulting break down of linear cell kill, for example due to saturation effects; however, we do not invoke this interpretation below.

\subsection{Model summary}

The healthy cell dynamics are governed by the equations for the $S, G_{2} / M, G_{0}$ and $G_{1}$ phases of the cell cycle, plus the chemotherapeutic damaged compartment and apoptotic cells, with further equations governing cell volume growth and the population volume. In summary, these are

$$
\begin{aligned}
\frac{\partial S}{\partial t}+\frac{\partial S}{\partial a}= & -\Phi S, \quad a \in\left[0, a_{2}\right], \\
\frac{\partial G_{\mathrm{m}}}{\partial t}+\frac{\partial G_{\mathrm{m}}}{\partial a}= & 0, \quad a \in\left[0, a_{3}\right], \\
\frac{\partial G_{0}}{\partial t}= & \mu\left(1-H_{0}\left[V(t)-V_{\max }\right]\right) G_{1}(v, t) \\
& -\xi\left(H_{0}\left[V(t-\Delta)-V_{\max }\right]\right) G_{0}(v, t-\Delta)-\zeta G_{0}(v, t), \quad v \in\left[v_{*}, 2 v_{*}\right] . \\
\frac{\partial G_{1}}{\partial t}+\dot{v} \frac{\partial G_{1}}{\partial v}= & \xi\left(H_{0}\left[V(t-\Delta)-V_{\max }\right]\right) G_{0}(v, t-\Delta)-\mu\left(1-H_{0}\left[V(t)-V_{\max }\right]\right) G_{1}(v, t) \\
& +2\left(1-H_{0}\left[V(t)-V_{\max }\right]\right) \delta\left(v-v_{*}\right) G_{\mathrm{m}}\left(a_{2}, t\right), \quad v \in\left[v_{*}, 2 v_{*}\right], \\
\frac{\partial S_{\mathrm{d}}}{\partial t}+\frac{\partial S_{\mathrm{d}}}{\partial a}= & \Phi S(a, t), \quad a \in\left[0, a_{2}\right], \\
\frac{d A}{d t}= & S_{\mathrm{d}}\left(a_{2}, t\right)-\chi A,
\end{aligned}
$$

with

$$
\begin{aligned}
V(t)= & \int_{\nu_{*}}^{2 v_{*}} v\left[G_{1}(v, t)+G_{0}(v, t)\right] \mathrm{d} v+2 v_{*} A(t) \\
& +2 v_{*}\left[\int_{0}^{a_{2}}\left\{S(a, t)+S_{\mathrm{d}}(a, t)\right\} \mathrm{d} a+\int_{0}^{a_{3}} G_{\mathrm{m}}(a, t) \mathrm{d} a\right],
\end{aligned}
$$




$$
\begin{aligned}
H_{0}\left[V(t)-V_{\max }\right] & = \begin{cases}0 & \text { if } V(t) \geqslant V_{\max }, \\
1 & \text { if } V(t)<V_{\max },\end{cases} \\
\dot{v} & =\left[v_{*} / a_{*}^{s}\right] H_{0}\left[V(t)-V_{\max }\right], \\
\delta\left(v-v_{*}\right) & \approx \begin{cases}\frac{1}{\epsilon_{*}} & v \in\left[v_{*}, v_{*}+\epsilon_{*}\right], \\
0 & \text { otherwise. }\end{cases}
\end{aligned}
$$

These are also subject to the boundary conditions of Section (2.1.6), whereby

$$
G_{\mathrm{m}}(0, t)=S\left(a_{2}, t\right), \quad S(0, t)=G_{1}\left(2 v_{*}, t\right) \dot{v}(t), \quad S_{\mathrm{d}}(0, t)=0,
$$

and

$$
\dot{v}(t) G_{1}\left(v_{*}, t\right)=2 G_{\mathrm{m}}\left(a_{3}, t\right) \quad \text { when } \quad \dot{v}>0 .
$$

The initial conditions are given by

$A(t)=S_{\mathrm{d}}(a, t)=0, \quad G_{0}(v, t)=G_{0}^{0}(v), \quad G_{1}(v, t)=G_{1}^{0}(v), \quad S(a, t)=S^{0}(a), \quad G_{\mathrm{m}}(a, t)=G_{\mathrm{m}}^{0}(a)$, for $t \in[-\Delta, 0]$, with the functions $G_{0}^{0}(v), G_{1}^{0}(v), S^{0}(a), G_{\mathrm{m}}^{0}(a)$ taken to be those arising from the large time asymptote of numerical simulations in the absence of chemotherapy.

The tumour cell population dynamics are governed by analogous cell cycle phase equations for cell densities, though subject to a distribution of $\mathrm{G}_{1}$ transit times; these equations are summarized by

$$
\begin{aligned}
\frac{\partial g_{1}}{\partial t}+\frac{\partial g_{1}}{\partial a} & =-\gamma(a) g_{1}, \quad a \in\left[0, a_{1}\right] \\
\frac{\partial s}{\partial t}+\frac{\partial s}{\partial a} & =-[\Phi+\alpha] s, \quad a \in\left[0, a_{2}\right] \\
\frac{\partial g_{\mathrm{m}}}{\partial t}+\frac{\partial g_{\mathrm{m}}}{\partial a} & =0, \quad a \in\left[0, a_{3}\right],
\end{aligned}
$$

with

$$
\begin{aligned}
& \gamma(a)=\frac{f(a)}{1-\int_{0}^{a} f\left(a^{\prime}\right) \mathrm{d} a^{\prime}}, \quad f(a)=N_{f} \exp \left(-\frac{\left(a-a_{*}^{c}\right)^{2}}{2 \sigma^{2}}\right), \\
& N_{f}^{-1}=\left[\int_{0}^{a_{1}} \mathrm{~d} a^{\prime} \exp \left(-\frac{\left(a^{\prime}-a_{*}^{c}\right)^{2}}{2 \sigma^{2}}\right)\right], \quad a \in\left[0, a_{1}\right] .
\end{aligned}
$$

The boundary conditions are

$$
g_{1}(0, t)=2 g_{\mathrm{m}}\left(a_{3}, t\right), \quad s(0, t)=\int_{0}^{a_{1}} \gamma\left(a^{\prime}\right) g_{1}\left(a^{\prime}, t\right) \mathrm{d} a^{\prime}, \quad g_{\mathrm{m}}(0, t)=s\left(a_{2}, t\right),
$$

with the initial distribution for $g_{1}, s$ and $m$ given by the large time asymptotic distribution of cells among the different cell cycle compartments predicted by the model in the absence of chemotherapy 
and an overall scaling determined by the total initial tumour cell number. The non-dimensionalization of these models, plus reference parameter values and their motivation are presented in the appendix.

The tumour and healthy cell dynamics are investigated in the context of chemotherapeutic protocols as follows. For a given set of parameters dictating cell behaviour, there are two remaining degrees of freedom governing the chemotherapeutic protocol given an implicit assumption of administering the maximally tolerated dose. In particular, once the duration of the drug administration and the rest phase of the protocol are prescribed, i.e. $t_{1}$ and $k t_{1}$, the intensity of the treatment, $\hat{m}$, is fixed by the maximally tolerated dose, denoted $m$, allowed by the toxicity constraint

$$
\frac{\int_{v_{*}}^{2 v_{*}} \mathrm{~d} \nu G_{0}(v, t)}{\int_{\nu_{*}}^{2 v_{*}} \mathrm{~d} \nu G_{0}^{\text {equilibrium }}(v)} \geqslant \Upsilon
$$

The cell kill function is then

$$
\Phi=m \phi(t)
$$

where $\phi(t)$ is the indicator function for the presence of drug given the protocol parameters, $t_{1}, k t_{1}$, as depicted in Fig. 3.

We can then simulate the tumour cell model with the resulting protocol to determine the prediction for the probability of success, which is equivalent to the probability that resistance does not emerge. This is given by

$$
p_{0}(\infty)=\exp \left[-\alpha \int_{0}^{\infty} \int_{0}^{a_{2}} s\left(a^{\prime}, t^{\prime}\right) \mathrm{d} a^{\prime} \mathrm{d} t^{\prime}\right],
$$

as deduced via (18) and numerically determined by calculating the long time asymptote of $p_{0}(t)$, with the chemotherapeutic protocol continuing throughout the simulation. In particular, for the numerical simulations below, it has been explicitly checked that $p_{0}(t)$ has asymptoted within tolerance bounds at the end of the simulation and thus can be interpreted $p_{0}(\infty)$, giving the model prediction for the probability of success; explicit examples in analogous models are presented by Gaffney (2004).

Within this framework, varying the duration of the drug administration and the rest phase, the influence of a toxicity constraint, cell cycle phase specificity and evolution of resistance on protocol schedulings with maximally tolerated dosages can be explored. An upwind finite-difference numerical scheme is utilized for the numerical solution of the partial differential equations, as required for explorations of the presented model. The presence of the time delay $\Delta$ entails that the algorithm stores a history of the system for a time $\Delta$; otherwise the numerical implementation is standard (Morton and Mayers, 2005).

\section{Results}

\subsection{Overview of figures}

The plots in Column I in each of Figs. 4-6 depict the maximally tolerated value of the cell kill area under curve, $\psi$, compatible with the toxicity constraint for a drug administration duration of $t_{1}$ and rest phase $k t_{1}$. This may be seen from the heat map presented within any given plot: the maximally tolerated value of $\psi$ for each value of $t_{1}$ and $k t_{1}$ can be obtained by comparing the colour of the plot at a given $\left(t_{1}, k t_{1}\right)$ coordinate to the colourbar on the right of the plot; analogous remarks apply for all plots presented. Note that it is particularly convenient to present the cell kill area under curve, $\psi$, as the measure of maximally tolerated administration intensity by virtue of the fact it is dimensionless, while 

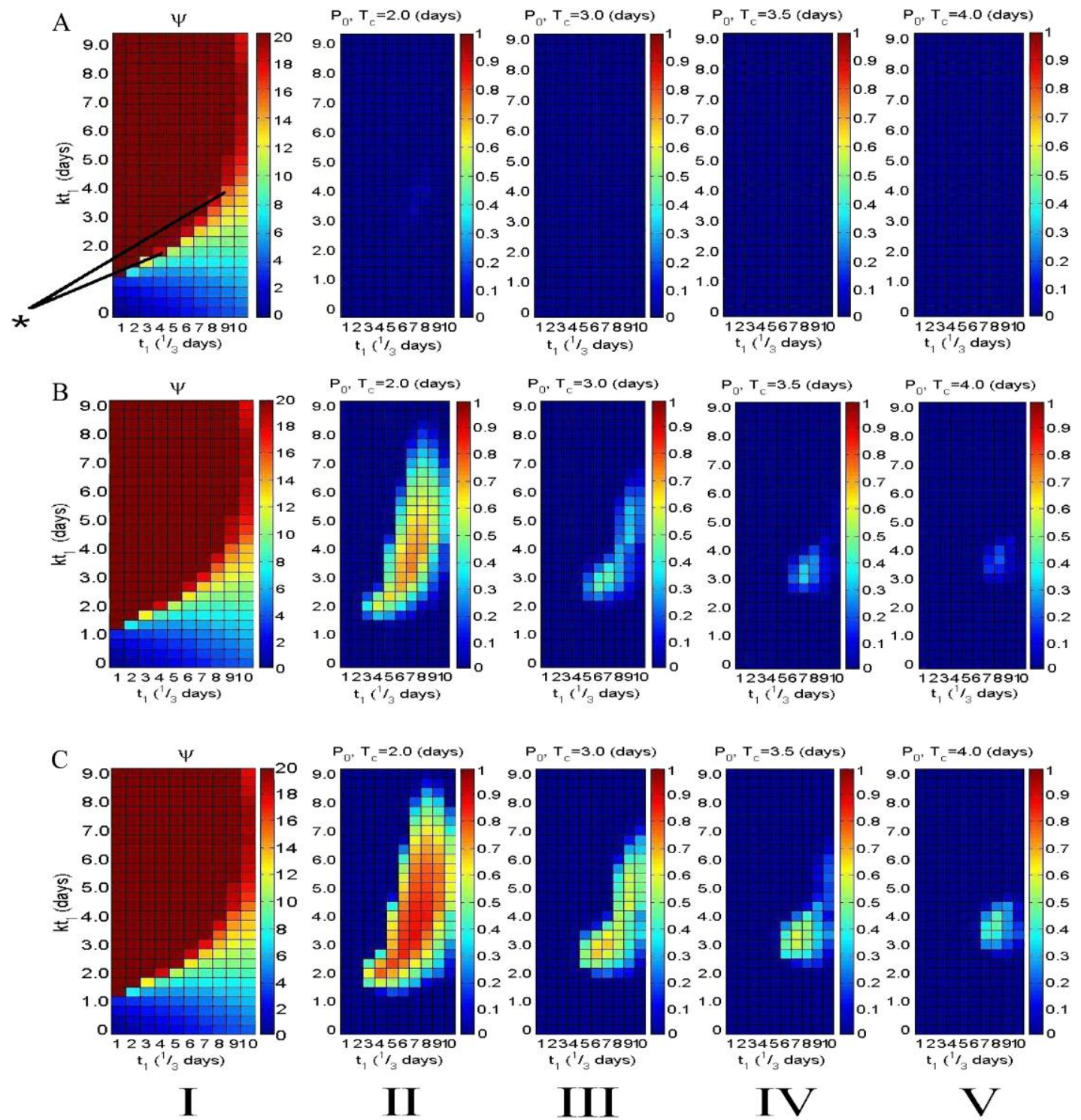

FIG. 4. Predictions for the probability of success given maximally tolerated dosages and alterations in the protocol scheduling; variations in the median tumour cell cycle time and the mutation rate to resistance are also considered. In the above plots, the drug administration time, $t_{1}$, is in the range $t_{1} \in\left[1 / 3\right.$ days, $10 / 3$ days], along the horizontal axis, while the rest phase, $k t_{1}$, is in the range $k t_{1} \in[0,9$ days $]$ along the vertical axis. Column I gives the maximally tolerated cell kill area under curve, $\psi$, compatible with the toxicity constraint for the corresponding protocol rest phase and drug administration duration. Note the transitional region in $\psi$, points on which are highlighted by the asterisk; see text for further details. In the remaining columns are plots for the probability of protocol success, $p_{0}(\infty)$, as parameter values are varied. All plots below are presented analogously. In Columns II-V, the parameter $\sigma^{2}$, which is a measure of the variance of the tumour cell cycle time, is given by $\sigma^{2} / T_{\mathrm{c}}^{2}=0.125 / 2$, where $T_{\mathrm{c}}$ denotes the median tumour cell cycle time. The median tumour cell cycle time, $T_{\mathrm{c}}$, is varied on moving between the second and fifth columns, taking the respective values of 2.0 days, 3.0 days, 3.5 days and 4.0 days. The mutation rate, $\alpha$, differs among Rows A-C, taking the respective values of $10^{-4}[\ln 2] / a_{2}, 10^{-6}[\ln 2] / a_{2}, 5 \times 10^{-7}[\ln 2] / a_{2}$, where $a_{2}$ is the S-phase duration. Further details on parameter estimation are presented in the appendix. 

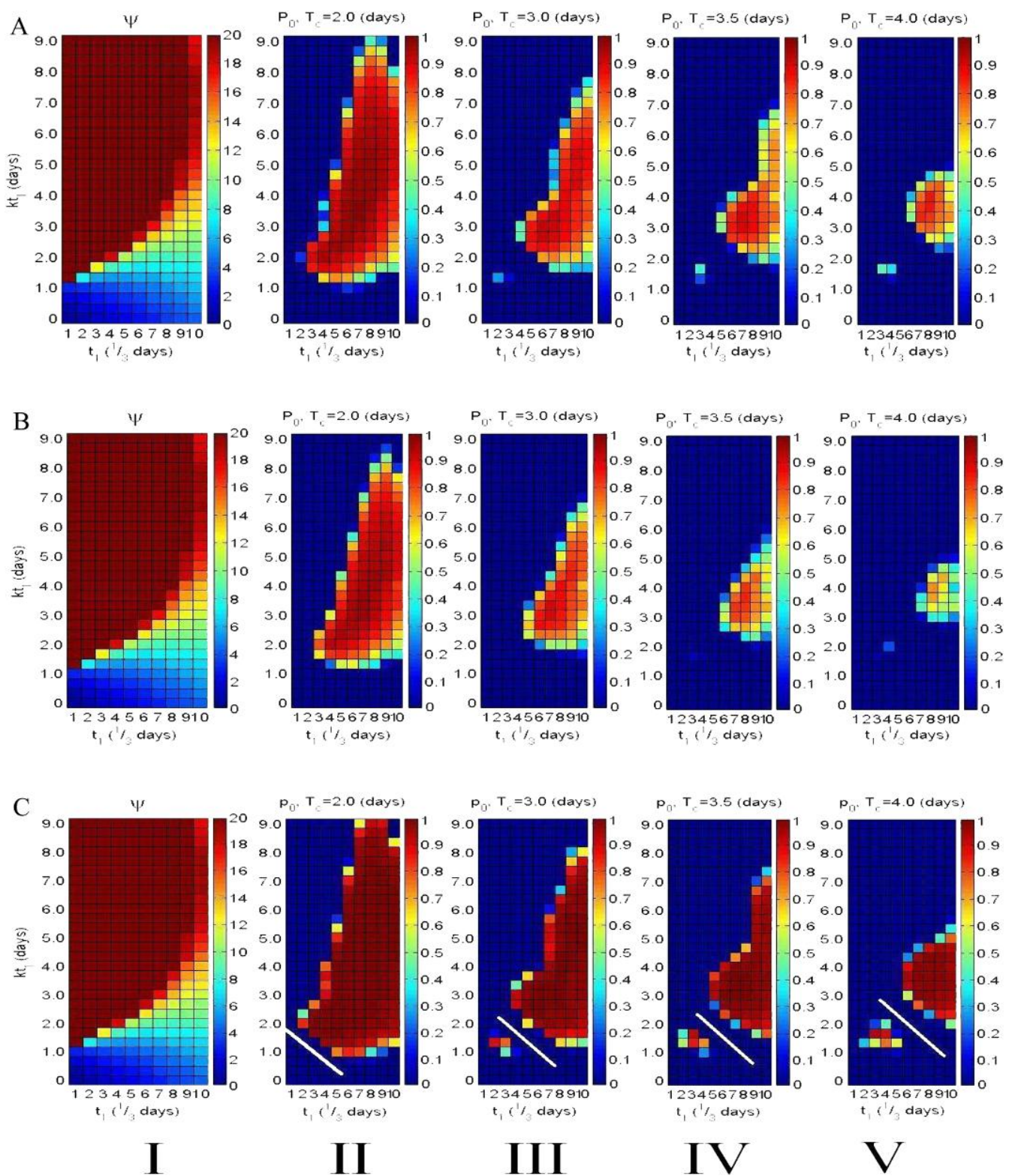

$p_{0} \cdot T_{r}=4.0$ (days)

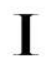

FIG. 5. Predictions for the probability of success given maximally tolerated dosages and alterations in the protocol scheduling; alterations in the median and variance of the tumour cell cycle time are also considered along with variations in the mutation rate to resistance. In the above plots, the drug administration time, $t_{1}$, is in the range $t_{1} \in[1 / 3$ days, $10 / 3$ days $]$, along the horizontal axis, while the rest phase, $k t_{1}$, is in the range $k t_{1} \in[0,9$ days $]$ along the vertical axis. Column I gives the maximally tolerated cell kill area under curve, $\psi$, compatible with the toxicity constraint for the corresponding protocol rest phase and drug administration duration. In Columns II-V are plots for the probability of protocol success, $p_{0}(\infty)$, as parameter values are varied. In the Rows A-C changes in the mutation rate, $\alpha$, and the variance of the tumour cell cycle time, as governed by $\sigma^{2}$, are explored. With $a_{2}, T_{\mathrm{c}}$, respectively, denoting the S-phase duration and the median tumour cell cycle time, these parameters are Row A: $\alpha=10^{-7}[\ln 2] / a_{2}, \sigma^{2}=0.125 T_{\mathrm{c}}^{2} / 2$; Row B: $\alpha=10^{-7}[\ln 2] / a_{2}, \sigma^{2}=0.25 T_{\mathrm{c}}^{2} / 2 ;$ Row C: $\alpha=10^{-8}[\ln 2] / a_{2}$, $\sigma^{2}=0.125 T_{\mathrm{c}}^{2} / 2$. The white lines in the plots of Row $\mathrm{C}$ are explained in the text. 

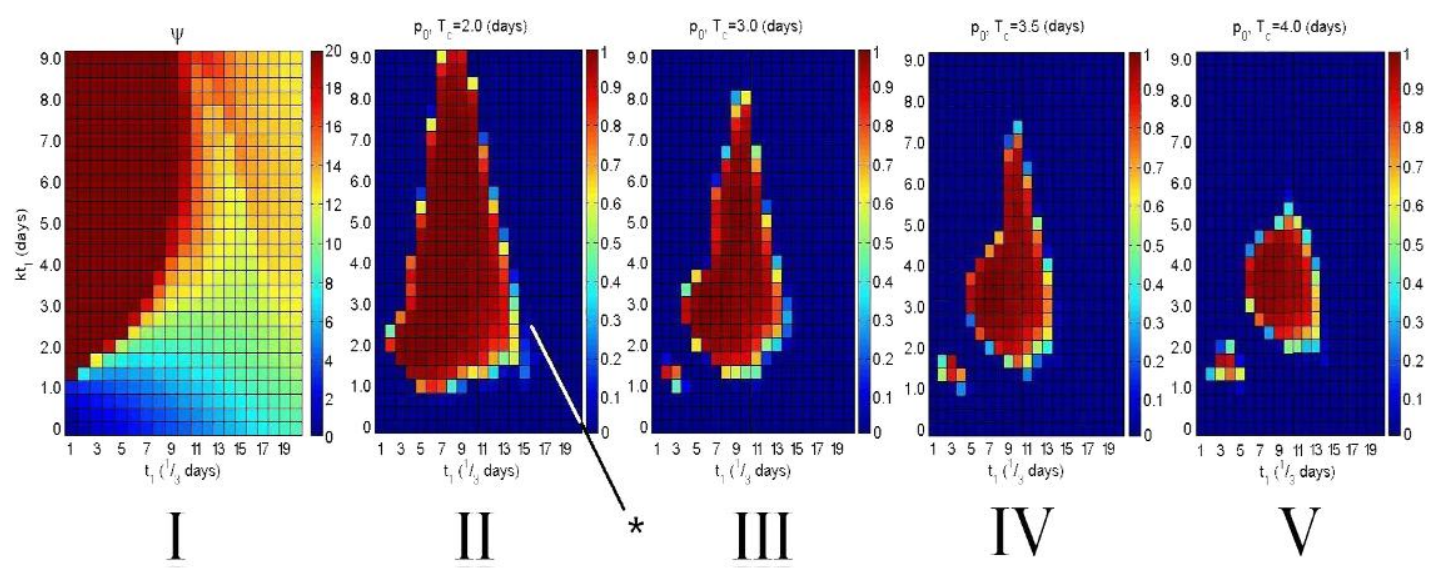

FIG. 6. Predictions for the probability of success given maximally tolerated dosages and alterations in the protocol scheduling; the drug administration time, $t_{1}$ has a larger range than considered previously with $t_{1} \in[1 / 3$ days, 20/3 days] along the horizontal axis. The rest phase, $k t_{1}$, is again in the range $k t_{1} \in[0,9$ days $]$ along the vertical axis. The first column gives the maximally tolerated cell kill area under curve, $\psi$, compatible with the toxicity constraint for the corresponding protocol rest phase and drug administration duration. In the remaining plots, the probability of protocol success, $p_{0}(\infty)$, is depicted as the median tumour cell cycle time, $T_{\mathrm{c}}$, is varied. The mutation rate, $\alpha$, is given by $\alpha=10^{-8}[\ln 2] / a_{2}$, and $\sigma^{2}$, which governs the variance of the tumour cell cycle time is given by $\sigma^{2}=0.125 T_{\mathrm{c}}^{2} / 2$. Note that the asterisk highlights the observation that sufficient increases in the drug administration time, $t_{1}$, away from the transitional region of maximally tolerated cell kill area under curve, $\psi$, results in a substantial reduction in protocol effectiveness.

the numerical simulations of the healthy cells on the one hand, and the tumour cells on the other, are based on different non-dimensionalizations for convenience, as detailed in the apendix.

In the remaining plots, in Columns II-V of Figs. 4-6, the probability of protocol success, $p_{0}(\infty)$, is depicted as the drug administration duration, $t_{1}$, is varied along the horizontal axis of each plot and the rest phase, $k t_{1}$, is varied along the vertical axis, given maximally tolerated dosages. The duration of the median tumour cell cycle time, $T_{\mathrm{c}}$, its variance plus the mutation rates to resistance are varied in these figures; all other parameters are derived from estimates presented in the appendix.

\subsection{Model predictions}

In Fig. 4, maximally tolerated cell kill area under curves, $\psi$, and the probability of protocol success, $p_{0}(\infty)$, are presented for different protocol schedulings as the median tumour cell cycle durations are varied between 2.0 days and 4.0 days in Columns II-V. In Rows A-C, mutation rates are varied from $10^{-4}[\ln 2] / a_{2}$ to $5 \times 10^{-7}[\ln 2] / a_{2}$, where $a_{2}$ is the $S$-phase duration.

Note that Row A demonstrates that for the extremely aggressive resistance mutation rate of $10^{-4}[\ln 2] / a_{2}$, there is effectively no protocol success. In Rows B, C of Fig. 4, and also in Figs. 5 and 6 results with less aggressive, but nonetheless realistic, mutation rates are presented.

In the plots presented in these figures, the regions where protocol success is observed have a boundary that is delimited towards the left by the loci of points where the maximally tolerated cell kill area under curve, $\psi$, changes very rapidly. This transitional region in the maximally tolerated cell kill area under curve is emphasized in Fig. 4A.I via the asterisk.

In addition, limited increases in the drug administration time, $t_{1}$, away from the boundary where $\psi$ changes rapidly also yield successful protocols, especially for intermediate values of the rest phase, $k t_{1}$. This can also be observed for the parameter values of Fig. 5, where lower mutation rates and higher tumour cell cycle time variances are considered; similar comments apply to Fig. 6 that explores an 
extended range of the drug administration time. In the latter, an asterisk emphasizes that very large increases in the drug administration time, $t_{1}$, are counter-productive. However, protocol failure can still be apparent in regions where the maximally tolerated cell kill area under curve changes very rapidly if the rest phase is either very high or very low; this can be readily observed in all of Figs. 4-6.

In Fig. 5, changes in the variance of the tumour cell cycle time are also explored. Consider Row $\mathrm{C}$; for relatively low rest phases a different, if subtle, behaviour emerges where a region of protocol failure occurs along the white line depicted in plots 5C.II-5C.V. This can also be observed in Fig. 6 and, to a lesser extent, in Row A of Fig. 5. The white lines in plots 5C.II-5C.V satisfy $t_{1}+k t_{1}=T_{c}$, where $T_{\mathrm{c}}$ is the median tumour cell cycle time, and Row $\mathrm{C}$ corresponds to a particularly low variance of the tumour cell cycle time. The fact the protocol is ineffective in this specific region, especially for low variances of the cell cycle time, evidences a resonant failure of the chemotherapeutic protocol; in particular, a fine tuning of parameters is required for the presentation of this mechanism.

From Figs. 4-6, one can also infer the effects of changing the tumour cell cycle time and its variance. By comparing Columns II, III, IV and V in any of the figures, where the median tumour cell cycle time is, respectively, 2.0 days, 3.0 days, 3.5 days and 4.0 days, one has that increases in the cell cycle time induces poorer outcomes. Similarly, by comparing Rows A and B of Fig. 5, one can see that increasing the tumour cell cycle variance also reduces the protocol effectiveness in general (away from regions where resonant failure may be important).

Note from Figs. 4-6 that reducing the rest phase from a very large value will either induce improvements in the outcome, at least initially or will have no effect. Thus, dose densification in general is predicted to often be beneficial but not universally so. In particular, aggressive dose densification eventually yields poor results once reductions in the rest phase result in extensive movement away from the transitional region where the maximal cell kill area under curve, $\psi$, changes rapidly. It can also be clearly observed that manipulating the dose administration time in addition to dose densification can yield substantial improvements when dose densification in isolation is ineffective.

\section{Discussion and conclusions}

In this study, we have constructed a model incorporating the response of tumour and healthy tissue subjected to a cell cycle phase-specific chemotherapeutic protocol. Motivated by 5-FU in the arena of colorectal adjuvant therapy, we have considered intermittently continuous administrations of an S-phase-specific drug with the aim of assessing how treatment toxicity and the prospect of resistance evolution may influence concepts for optimizing protocol scheduling. In particular, the focus has been on the effects of dose densification and alterations in drug administration duration for schedules characterized by maximally tolerated dosages.

Unsurprisingly, for sufficiently aggressive evolution to resistance the model predicts that treatment is ineffective. As necessary for confidence in the modelling predictions, given the difficulties with drug resistance in practice, this happens easily within the empirical bounds on mutation rates.

For lower mutation rates, though again in the empirical range, the optimal protocol scheduling parameters are delimited on the left in Figs. 4-6 by the region where the tolerated dosage changes sharply. We refer to this as the region of transitional dosage. The fact such sharp changes exist leads to a model prediction that, in general, one can expect sensitivity to protocol changes.

Effective schedulings, if they exist, appear in the transitional dosage region and for limited increases in the drug administration time, $t_{1}$, away from this region. The fact the region of transitional dosage delimits the regions of effective scheduling demonstrates the modelling prediction that toxicity limitations are a major factor dictating protocol outcomes, even when maximally tolerated doses are 
considered. In particular, once the scheduling timings are sufficiently relaxed to allow extensive intensity escalation before attaining the maximally tolerated dosage, poor outcomes are predicted. In addition, if drug administrations are for greatly extended periods, the resulting restrictions in drug intensity again yield predictions of poor outcomes.

In contrast, intermediate timings characterize effective protocols and are governed by the level of escalation allowed, that is the level of toxicity present. This also demonstrates rest phases are predicted to be critical in designing effective protocols using maximally tolerated doses, despite the consequences of partial tumour tissue recovery.

However, not all schedulings in regions of transitional dosage are predicted to be effective, highlighting that additional aspects of the biology can be important. At high values of the drug rest phase, $k t_{1}$, within the transitional dosage region, this failure is anticipated to be a result of extensive tumour tissue recovery; note also that in this region of parameter space one has the rest phase is substantially greater than the drug administration duration. For very short rest phases within the transitional dosage region, tolerable administration durations are even shorter so that once more the rest phase is substantially longer than the drug administration duration and, in effect, the majority of the protocol is in a rest phase, allowing tumour recovery.

Cell cycle resonance effects are also observed illustrating one aspect of how the cell cycle can influence the model predictions. In particular, protocol choices with a period equal to the median tumour cell cycle time yield an uncharacteristically poor outcome, especially for smaller variances of the tumour cell cycle time. For such schedulings, the majority of tumour cells are protected by the fact the period of the protocol scheduling is in synchrony with the tumour cell cycle time, which does not vary extensively between cells when there is a low variance of the tumour cell cycle duration. In particular, after the initial elimination of the S-phase cells by the first drug administration, remaining tumour cells are not extensively killed as the protocol is always applied at the same point of the cell cycle, while the remaining tumour cells are typically not in S-phase during the administrations. Although this resonant failure is not universally seen in the modelling, as it is sensitive to parameter values, it nonetheless advocates a prediction that resonances between protocol timings and the tumour cell cycle have the potential to reduce therapeutic effectiveness for cell cycle phase specific interventions.

Apart from the possibility of resonance, it is observed to be generally more difficult to eliminate a tumour which is growing more slowly or which possesses a greater heterogeneity in its cell cycle time. Slower growth entails that fewer tumour cells are susceptible at any given time within the model, leading to a larger tumour cell sanctuary due to cell cycle effects, highlighting an important consequence of the cell cycle on modelling predictions.

Nonetheless, alterations in the tumour cell cycle time or its variance do not impact upon the observations of the importance of the transitional region in predictions of a successful outcome. The same observation is true over a large range of mutation rates even though mutation to resistance has an especially profound effect on the overall chance of success. Consequently, we see that as parameters are altered, the quantitative details of the probability of success do change, often extensively. However, the importance of the region of transitional dosage in locating relative protocol improvements is retained for any such parameter variations, at least providing extremely small or extremely large rest phases are avoided, along with resonant values of the period of the protocol. Furthermore, while it is not feasible to consider all of parameter space such observations have also emerged for numerous other plausible parameter possibilities (Boston, 2008). Thus, modelling predictions for locating effective scheduling protocols apply for very large ranges of parameters, even if the quantitative details differ substantially.

We now explicitly consider dose densification. For a given dose administration duration with a very large rest phase, the predicted outcome is poor. Subsequently reducing the rest phase, while maintaining 
maximally tolerable dosings, is predicted to either have no effect or to lead to initial improvements, with subsequent rest phase reductions eventually leading to relatively poor outcomes. Thus, dose densification is not predicted to be universally beneficial. Furthermore, even if it does yield improvements, very aggressive dose densification is not advocated by the modelling predictions; this is already recognized as 'not trying to kill the [tumour] cells that are already dead' (Norton, 2005). In particular, once the dose densification starts inducing substantial decreases in the maximally tolerated dose, sufficiently aggressive further dose densing is predicted to be counter-productive.

These observations nonetheless indicate that dose densification is very useful in the current context even though theoretical framework developed by Norton (1998a,b, 2001) does not explicitly model resistance, the cell cycle or toxicity. However, our results additionally highlight the importance of varying the drug administration duration, especially if dose densing in isolation is ineffective. It is also interesting to note that positive results are observed for general protocol escalations, with variations in the drug administration time, for Phase-I trials, assessing safety profiles, and for mouse models, though currently for systems with non-trivial pharmacokinetics (Kolinsky et al., 2009; Traina et al., 2008) rather than infusional adjuvant therapies.

Finally, one should note that the conclusions drawn are based on a model of infusional treatments with single agent 5-FU for freshly vascularized nodules. It is therefore of course important to consider whether generalizations of this modelling framework further influence the conclusions. Thus, future studies will also consider the influence of combination therapies, non-exponential growth dynamics, non-trivial pharmacokinetics and differences between regional and systemic treatments.

\section{Acknowledgement}

We are grateful to Prof. D.J. Kerr for stimulating discussions.

\section{Funding}

This publication is based on work supported in part by Award No. KUK-C1-013-04, made by King Abdullah University of Science and Technology (KAUST). It was also supported in part by the Engineering and Physical Sciences Research Council, UK (EPSRC GR/S72023/01).

\section{REFERENCES}

Abdreeff, M., Goodrich, D. W. \& Pardee, A. B. (2000) Cell proliferation, differentiation and apoptosis, Chapter 2. The Holland-Frei Cancer Medicine, 5th edn. (R. C. Bast, D. W. Kufe, R. E. Pollack, R. R. Weischelbaum, J. F. Holland \& E. Frei eds). Hamilton, ON: BC Decker Inc., pp. 17-32.

ADAM, R. (2006) Current surgical strategies for the treatment of colorectal cancer liver metastases. Eur. J. Cancer Suppl., 2, 21-26.

AfENYA, E. K. (2001) Recovery of normal hemopoiesis in disseminated cancer therapy-a model. Math. Biosci., 172, 15-32.

AgUR, Z., ARnON, R. \& SCHECHTER, B. (1988) Reduction of cytotoxicity to normal tissues by new regimens of cell-cycle phase-specific drugs. Math. Biosci., 92, 1-15.

Agur, Z., ARnon, R. \& SCHECHTER, B. (1992) Effect of the dosing interval on myelotoxicity and survival in mice treated by cytarabine. Eur. J. Cancer, 28A, 1085-1090.

BARBolosi, D. \& ILIADIS, A. (2001) Optimizing drug regimens in cancer chemotherapy: a simulation study using a PK-PD model. Comput. Biol. Med., 31, 157-172.

Boston, E. (2008) Effects of incorporating toxic constraints to predictions of optimal chemotherapy scheduling. Ph.D Thesis, University of Birmingham, UK. 
Browder, T., Butterfield, C. E., Kraling, B. M., Shi, B., Marshall, B., OReilly, M. \& Folkman, J. (2000) Antiangiogenic scheduling of chemotherapy improves efficacy against experimental drug-resistant cancer. Cancer Res., 60, 1878-1886.

Chiorino, G., Metz, J. A. J., Tomasoni, D. \& Ubezio, P. (2001) Desynchronization rate in cell populations: mathematical modeling and experimental data. J. Theor. Biol., 208, 185-199.

Citron, M., Berry, D., Cirrincione, C., Carpenter, J., Hudis, C., Gradishar, W., Davidson, N., Ingle, J., Martino S., Livingston, R., Winer, E., Muss, H. \& Norton, L. (2002) Superiority of dose-dense (DD) over conventional scheduling (CS) and equivalence of sequential (SC) vs. combination adjuvant chemotherapy (CC) for node-positive breast cancer (CALGB 9741, INT C9741). Breast Cancer Research and Treatment 76, S32-S32.

Citron, M. L., Berry, D. A., Cirrincione, C., Hudis, C., Winer, E. P., Gradishar, W. J., Davidson, N. E., Martino, S., Livingston, R., Ingle, J. N., Perez, E. A., Carpenter, J., Hurd, D., Holland, J. F., Smith, B. L., Sartor, C. I., Leung, E. H., Abrams, J., Schilsky, R. L., Muss, H. B. \& NORTON, L. (2003) Randomized trial of dose-dense versus conventionally scheduled and sequential versus concurrent combination chemotherapy as postoperative adjuvant treatment of node-positive primary breast cancer: first report of intergroup trial C9741/cancer and leukemia group b trial 9741. J. Clin. Oncol., 21, $1431-1439$.

Cohen, A. D. \& Kemeny, N. (2003) An update on hepatic arterial infusion chemotherapy for colorectal cancer. Oncologist, 8, 553-556.

COJOCARU, L. \& AGUR, Z. (1992) Theoretical analysis of interval drug dosing for cell-cycle-phase-specific drugs. Math. Biosci., 109, 85-97.

Conlon, I. \& RAFF, M. (2003) Differences in the way a mammalian cell and yeast cells coordinate cell growth and cell-cycle progression. J. Biol., $2,7$.

CoOper, S. (2000) The continuum model and the G1-control of the mammalian cell cycle. Progress in Cell Cycle Research (M. Meijer, A. Jezequel \& B. Ducommun, eds.), vol. 4, New York: Plenum Press, pp. 27-39.

Costa, M. I. S., Boldrini, J. L. \& BAssanezi, R. C. (1995) Chemotherapeutic treatments involving drug resistance and level of normal cells as a criterion of toxicity. Math. Biosci., 125, 211-228.

Court, F. G., Wemyss-Holden, S. A., Dennison, A. R. \& Maddern, G. J. (2002) The mystery of liver regeneration. Br. J. Surg., 89, 1089-1095.

Deplacido, S., Perrone, F., Carlomagno, C., Morabito, A., Pagliarulo, C., Lauria, R., Marinelli, A., Delaurentis, M., Varriale, E., Petrella, G., Gallo, C. \& Bianco, A. R. (1995) $\mathrm{CMF}$ vs alternating $\mathrm{CMF} / \mathrm{EV}$ in the adjuvant treatment of operable breast cancer. a single centre randomised clinical trial (Naples GUN-3 study). Br. J. Cancer, 71, 1283-1287.

Dibrov, B. (1998) Resonance effect in self-renewing tissues. J. Theor. Biol., 192, 15-33.

Ensminger, W., Rosowsky, A., Raso, V., Levin, D., Glode, M., Cone, S., Steele, G. \& Frei, E. (1978) A clinical-pharmacological evaluation of hepatic arterial infusions of 5 fluoro-2/deoxuridine and 5-fluorouracil. Ann. Oncol., 38, 3784-3792.

Fallik, D., Ychou, M., Jacob, J., Colin, P., Seitz, J. F., Baulieux, J., Adenis, A. \& Douillard, J. (2003) Hepatic arterial infusion using pirarubicin combined with systemic chemotherapy: a phase II study in patients with nonresectable liver metastases from colorectal cancer. Ann. Oncol., 14, 856-863.

Frei, E. \& Antman, K. (2000) Principles of dose, schedule and combination chemotherapy, Chapter 40. The Holland-Frei Cancer Medicine, 5th edn. BC Decker Inc., pp. 556-568.

GAFFNEY, E. A. (2004) The application of mathematical modelling to aspects of adjuvant chemotherapy scheduling. J. Math. Biol., 48, 375-422.

GAFFNEY, E. A. (2005) The mathematical modelling of adjuvant chemotherapy scheduling: incorporating the effects of protocol rest phases and pharmacokinetics. Bull. Math. Biol., 67, 563-611.

Goldie, J. H. \& Coldman, A. J. (1979) A mathematical model for relating the drug sensitivity of tumours to their spontaneous mutation rate. Cancer Treat. Rep., 63, 1727-1731. 
Goldie, J. H. \& Coldman, A. J. (1983a) A model for the resistance of tumor cells to cancer chemotherapy. Math. Biosci., 65, 291-307.

Goldie, J. H. \& Coldman, A. J. (1983b) Quantitative model for multiple levels of drug resistance in clinical tumours. Cancer Treat. Rep., 67, 923-931.

Goldie, J. H. \& Coldman, A. J. (1986) Application of theoretical models to chemotherapy protocol design. Cancer Treat. Rep., 70, 127-131.

Goldie, J. H. \& Coldman, A. J. (1998) Drug Resistance in Cancer: Mechanisms and Models. Cambridge: Cambridge University Press.

Goldie, J. H., Coldman, A. J. \& Gudauskas, G. A. (1982) Rationale for the use of alternating non-cross resistant chemotherapy. Cancer Treat. Rep., 66, 439-449.

Hahnfeldt, P., Folkmany, J. \& Hlatky, L. (2003) Minimizing long-term tumor burden: the logic for metronomic chemotherapeutic dosing and its antiangiogenic basis. J. Theor. Biol., 220, 545-554.

HAINES, I. E. (2008) Dose selection in phase I studies: why we should always go for the most effective (correspondence). J. Clin. Oncol., 26, 3650-2652.

InOMATA, A., HoRII, I. \& SUZUKI, K. (2002) 5-Fluorouracil-induced intestinal toxicity: what determines the severity of damage to murine intestinal crypt epithelia? Toxicol. Lett., 133, 231-240.

Jaffrezou, J. P., Chen, G., Duran, G. E., Kuhl, J. S. \& Sikic, B. (1994) Mutation rates and mechanisms of resistance to etoposide determined from fluctuation analysis. J. Natl. Cancer Inst., 86, 1152-1158.

Kemeny, N. \& FATA, F. (2001) Hepatic-arterial chemotherapy. Lancet Oncol., 2, 418-428.

Kemeny, N., Huang, Y., Cohen, A., Shi, W., Conti, J., Brennan, M., Bertino, J., Turnbull, A., Sullivan, D., Stockman, J., Blumgart, L. \& Fong, Y. (1999) Hepatic arterial infusion of chemotherapy after resection of hepatic metastases from colorectal cancer. N. Engl. J. Med., 341, 2039-2048.

Kolinsky, K., Zhang, Y., Dugan, U., Heimbrook, D., Packman, K. \& Higgins, B. (2009) Novel regimens of capecitabine alone and combined with irinotecan and bevacizumab in colorectal cancer xenografts. Anticancer Res., 29, 91-98.

LAW, L. W. (1952) Origin of the resistance of leukaemic cells to folic acid antagonists. Nature, 169, 628-629.

LeSage, G., Glaser, S. S., Gubba, S., Robertson, W. E., Phinizy, J., Lasater, J., Rodgers, R. D. \& ALPINI, G. (1996) Regrowth of the rat biliary tree after 70\% partial hepatectomy is coupled to increased secretin-induced ductal secretion. Gastroenterology, 111, 1633-1644.

Loeb, L. A., Loeb, K. R. \& Anderson, J. P. (2003) Multiple mutations and cancer. Proc. Natl. Acad. Sci. USA., 100, 776-781.

LURIA, S. E. \& DELBRÜCK, M. (1943) Mutations of bacteria from virus sensitivity to virus resistance. Genetics, 28, 491-511.

MARKMAN, M. (1993) Optimal versus maximally tolerated dose in cancer chemotherapy treatment. J. Cancer Res. Clin. Oncol., 119, 576-577.

Marzioni, M., Glaser, S., Francis, H., Marucci, L., Benedetti, A., Alvaro, D., Taffetani, S., Ueno, Y., Roskams, T., Phinizy, J. L., Venter, J., Fava, G., Lesage, G. D. \& Alpini, G. (2005) Autocrine/paracrine regulation of the growth of the biliary tree by the neuroendocrine hormone serotonin. Gastroenterology, 128, 121-137.

Matveev, A. S. \& SAVKin, A. V. (2005) Influence of tumours on normal cells and optimal chemotherapy regimens: the case of several drugs and toxicity constraints. Math. Med. Biol., 22, 143-162.

Midgley, R. S. J. \& KERR, D. J. (1999) Seminar in colorectal cancer. Lancet, 353, 391-399.

Midgley, R. S. J. \& KeRR, D. J. (2000) ABC of colorectal cancer: adjuvant therapy. BMJ, 321, 1208-1211.

Morton, K. \& MaYers, D. (2005) Numerical Solution of Partial Differential Equations: An Introduction. Cambridge: Cambridge University Press.

Musgrove, E. A., Lee, C. S. L., Buckley, M. F. \& Sutherland, R. L. (1994) Cyclin D1 induction in breast cancer cells shortens G1 and is sufficient for cells arrested in G1 to complete the cell cycle. Proc. Natl. Acad. Sci. USA., 91, 8022-8026. 
Nicum, S., Midgley, R. \& KerR, D. J. (2000) Chemotherapy for colorectal cancer. J. R. Soc. Med., 93, 416419.

Norton, L. (1998a) A Gompertzian model of human breast cancer growth. Cancer Res., 48, 7067-7071.

Norton, L. (1998b) Evolving concepts in the systemic drug therapy of breast cancer. Semin. Oncol., 24, S103-S10-10.

Norton, L. (2001) Theoretical concepts and the emerging role of taxanes in adjuvant therapy. Oncologist, 6, 30-35.

NORTON, L. (2005) Conceptual and practical implications of breast tissue geometry: toward a more effective, less toxic therapy. Oncologist, 10, 370-381.

Norton, L. (2008) The concept of mathematically optimised dose-scheduling as applied to the adjuvant chemotherapy of primary breast cancer: theory and recent results. Eur. J. Cancer Suppl., 6, 10-16.

Norton, L. \& SimON, R. (1977) Tumor size, sensitivity to therapy, and design of treatment schedules. Cancer Treat. Rep., 61, 1307-1317.

Norton, L. \& Simon, R. (1986) The Norton-Simon hypothesis revisited. Cancer Treat. Rep., 70, 163-169.

Oкамото, S., SAKAI, M., UChidA, J. \& SAIto, H. (1996) 5-Fluorouracil induces apoptotic cell death with $\mathrm{G}_{2}$ phase arrest in human breast cancer grafted in nude mice. Anticancer Res., 16, 2699-2704.

PAlmes, D. \& SPIEgEL, H. (2004) Animal models of liver regeneration. Biomaterials, 25, 1601-1611.

PAnetTA, J. C. \& ADAM, J. (1995) A mathematical model of cycle-specific chemotherapy. Math. Comput. Modelling, 22, 67-82.

Piccart, M. J., Biganzoli, L. \& Di Leo, A. (2000) The impact of chemotherapy dose density and dose intensity on breast cancer outcome: what have we learned? Eur. J. Cancer, 36, S4-S10.

Rew, D. A. \& Wilson, G. D. (2000) Cell production rates in human tissue and tumours and their significance. Part II: clinical data. Eur. J. Surg. Oncol., 26, 405-417.

Sieber, M., Tesch, H., Pfistner, B., Rueffer, U., lathan, B., Brosteanu, O., Paulus, U., Koch, T., Pfreundschuh, M., Loeffler, M., Engert, A., Josting, A., Wolf, J., Hasenclever, D., FRANKLIN, J., et al. (2002) Rapidly alternating COPP/ABV/IMEP is not superior to conventional alternating COPP/ABVD in combination with extended-field radiotherapy in intermediate-stage hodgkin's lymphoma: final results of the German Hodgkin's Lymphoma study Group Trial HD5. J. Clin. Oncol., 20, 476-484.

Simon, R. \& NoRTON, L. (2006) The Norton-Simon hypothesis: designing more effective and less toxic chemotherapeutic regimens. Nat. Clin. Pract. Oncol., 3, 406-407.

Siodlak, M. Z., Stell, P. M., Wilson, J. A., Green, R. S., Allison, D., Adler, M. \& SquadrelliSARACENO, (1990) Alternating cisplatinum and VAC ineffective in end stage squamous cell carcinoma of the head and neck. J. Laryngol. Otol., 104, 631-633.

SKIPPER, H. E. (1965) The effects of chemotherapy on the kinetics of leukaemic cell behaviour. Cancer Res., $\mathbf{2 5 ,}$ $1544-1550$.

SkitzKi, J. \& ChANG, A. E. (2002) Hepatic artery chemotherapy for colorectal liver metastases: technical considerations and review of clinical trials. Surg. Oncol., 11, 123-135.

Summers, W. P. \& HANDSHUMACHER, R. (1973) The rate of mutation of 15178y asparagine-dependent mouse leukemia cells to asparagine independence and its biological consequences. Cancer Res., 33, 1775-1779.

Takahashi, T., Bhide, P. G., Goto, T., Miyama, S. \& Caviness, V. S. (1999) Proliferative behavior of the murine cerebral wall in tissue culture: cell cycle kinetics and checkpoints. Exper. Neurol., 156, 407-417.

Tebbutt, N. C., Cattell, E., Midgley, R., Cunningham, D. \& Kerr, D. (2002) Systemic treatment of colorectal cancer. Eur. J. Cancer, 38, 1000-1015.

Traina, T. A., Theodoulou, M., Feigin, K., Patil, S., Tan, K. L., Edwards, C., Dugan, U., NoRTON, L. \& HUdis, C. (2008) Phase I study of a novel capecitabine schedule based on the Norton-Simon mathematical model in patients with metastatic breast cancer. J. Clin. Oncol., 26, 1797-1802.

Ubezio, P., Tagliabue, G., Schechter, B. \& Agur, Z. (1994) Increasing 1-beta-d-arabinofuranosylcytosine efficacy by scheduled dosing intervals based on direct measurements of bone-marrow cell-kinetics. Cancer Res., 54, 6446-6451. 
WebB, G. (1990) Resonance phenomena in cell population chemotherapy models. Rocky Mountain J. Math., 20, $1195-1216$.

WheLdon, T. E. (1988) Mathematical Models in Cancer Research. Bristol: Adam Hilger.

WolfE, S. L. (1995) Introduction to Cell and Molecular Biology. California: Wadsworth.

Zetterberg, A., Larsson, O. \& Wiman, K. G. (1995) What is the restriction point? Curr. Opin. Cell Biol., 7, 835-842.

\section{Appendix}

\section{A.1. Non-dimensionalization}

The fact that the healthy and tumour cell population models decouple except for the toxicity constraint allows different non-dimensionalizations for each model; these are listed in Tables A1, A2. While we present results in terms of dimensional variables, the underlying numerical simulations explicitly consider the non-dimensionalized models and thus correspond to a family of solutions on varying the parameters governing the scalings within the non-dimensionalizations.

TABLE A1 The non-dimensionalization for the healthy cell population model. Note that $m$ is the maximum value of the drug cell intensity, $\hat{m}$, allowed by the toxicity constraint with an analogous relation between $\psi$ and $\hat{\psi}$. The non-dimensionalization for $\hat{m}$ and $\hat{\psi}$ is the same as for $m$ and $\psi$. Similarly, the non-dimensionalization for the initial condition functions $G_{1}^{0}, G_{0}^{0}, S^{0}$ and $G_{\mathrm{m}}^{0}$ in (11) is inherited from the non-dimensionalization of $G_{1}, G_{0}, S$ and $G_{\mathrm{m}}$

\begin{tabular}{lccc}
\hline Independent variables & $t \rightarrow \bar{t}=t / T_{s}$ & $a \rightarrow \bar{a}=a / T_{s}$ & $v \rightarrow \bar{v}=v / \nu_{*}$ \\
\hline Dependent variables & $G_{1} \rightarrow \bar{G}_{1}=v_{*} G_{1} / N_{s}$ & $S \rightarrow \bar{S}=T_{s} S / N_{s}$ & $G_{\mathrm{m}} \rightarrow \bar{G}_{\mathrm{m}}=T_{s} G_{\mathrm{m}} / N_{s}$ \\
& $G_{0} \rightarrow \bar{G}_{0}=v_{*} G_{0} / N_{s}$ & $S_{\mathrm{d}} \rightarrow \bar{S}_{\mathrm{d}}=T_{s} S_{\mathrm{d}} / N_{s}$ & $A \rightarrow \bar{A}=A / N_{s}$ \\
$V$ & $\rightarrow \bar{V}=V / \nu_{*} N_{s}$ & $\dot{v} \rightarrow \overline{\bar{v}}=T_{s} \dot{v} / \nu_{*}$ & \\
Parameters & $\mu \rightarrow \bar{\mu}=T_{s} \mu$ & $\xi \rightarrow \bar{\xi}=T_{s} \xi$ & $\zeta \rightarrow \bar{\zeta}=T_{s} \zeta$ \\
$\chi$ & $\rightarrow \bar{\chi}=T_{s} \chi$ & $\Phi \rightarrow \bar{\Phi}=T_{s} \Phi$ & $\Delta \rightarrow \bar{\Delta}=\Delta / T_{s}$ \\
$a_{*}^{s} \rightarrow \bar{a}_{*}^{s}=a_{*}^{s} / T_{s}$ & $a_{2} \rightarrow \bar{a}_{2}=a_{2} / T_{s}$ & $a_{3} \rightarrow \bar{a}_{3}=a_{3} / T_{s}$ \\
$V_{\max } \rightarrow \bar{V}_{\max }=V_{\max } / \nu_{*} N_{s}$ & $t_{1} \rightarrow \bar{t}_{1}=t_{1} / T_{s}$ & $k t_{1} \rightarrow \overline{k t}_{1}=k t_{1} / T_{s}$ \\
& $m \rightarrow \bar{m}=m T_{s}$ & $\psi \rightarrow \bar{\psi}=\psi$ & $\Upsilon \rightarrow \bar{\Upsilon}=\Upsilon$ \\
\hline
\end{tabular}

TABLE A2 The non-dimensionalization used for the cancerous cell population, with $k \in\{1,2,3\}$ for the subscript associated with the variables $a_{k}, \tilde{a}_{k}$. The non-dimensionalization for $\tilde{m}$ and $\tilde{\psi}$ is the same as for $m$ and $\psi$

\begin{tabular}{lccc}
\hline Independent variables & $t \rightarrow \tilde{t}=t / T_{\mathrm{c}}$ & $a \rightarrow \tilde{a}=a / T_{\mathrm{c}}$ & \\
\hline Dependent variables & $g_{1} \rightarrow \tilde{g}_{1}=T_{\mathrm{c}} g_{1} / N_{0}$ & $s \rightarrow \tilde{s}=T_{\mathrm{c}} s / N_{0}$ & $g_{\mathrm{m}} \rightarrow \tilde{g}_{\mathrm{m}}=T_{\mathrm{c}} g_{\mathrm{m}} / N_{0}$ \\
& $p_{0} \rightarrow \tilde{p}_{0}=p_{0}$ & & \\
Parameters & $\sigma \rightarrow \tilde{\sigma}=\sigma / T_{\mathrm{c}}$ & $a_{k} \rightarrow \tilde{a}_{k}=a_{k} / T_{\mathrm{c}}$ & $a_{*}^{c} \rightarrow \tilde{a}_{*}^{c}=a_{*}^{c} / T_{\mathrm{c}}$ \\
& $\gamma \rightarrow \tilde{\gamma}=T_{\mathrm{c}}$ & $\alpha \rightarrow \tilde{\alpha}=T_{\mathrm{c}} \alpha$ & $\Phi \rightarrow \tilde{\Phi}=T_{\mathrm{c}} \Phi$ \\
& $m \rightarrow \tilde{m}=T_{\mathrm{c}} m$ & $\gamma \rightarrow \tilde{\Upsilon}=\Upsilon$ & $f \rightarrow \tilde{f}=T_{\mathrm{c}} f$ \\
& $t_{1} \rightarrow \tilde{t}_{1}=t_{1} / T_{\mathrm{c}}$ & $k t_{1} \rightarrow \tilde{k} t_{1}=k t_{1} / T_{\mathrm{c}}$ & $\psi \rightarrow \tilde{\psi}=\psi$ \\
\hline
\end{tabular}




\section{A.2 Parameter estimation}

A.2.1 Healthy cell model parameters. The limiting volume of the healthy cell population, $V_{\max }$, is the number of toxicity limiting cells multiplied by the mean cell volume at equilibrium. With a fixed value for $N_{s}$, of the order of the number of toxicity limiting cells, we take

$$
V_{\max }=2 N_{s} \nu_{*} \stackrel{\text { def }}{=} \bar{V}_{\max } N_{s} \nu_{*}
$$

where $v_{*}$ is the cell volume after mitosis and $\bar{V}_{\max }$ is the non-dimensionalized limiting volume. The non-dimensionalized healthy cell model evolves from the initial conditions to an attracting basin where $\bar{V}_{\text {max }}=2$ and an equilibrium distribution among the cell cycle compartments; only at this point is the chemotherapeutic protocol considered. Note that if the initial conditions are taken to scale with $N_{s}$ too, the linearity of the healthy cell model ensures that non-dimensionalized model does not depend on $N_{s}$; this parameter simply governs the scale of the number of cells in the toxicity limiting population and an explicit estimate is not required. Linearity similarly ensures that the distribution of cells among the cell cycle compartments at equilibrium in the non-dimensionalized healthy cell model is not affected by the cell volume $v_{*}$.

For cycling healthy cells, the sum of the transit times in each of the cell cycle compartments under conditions of maximal growth is given by $a_{*}^{s}+a_{2}+a_{3}$; this is equal to $T_{s}$, the median cell cycle duration under such conditions. Takahashi et al. (1999) and Abdreeff et al. (2000) report that the S-phase lasts for about $12 \mathrm{~h}$ and that $\mathrm{G}_{2} / \mathrm{M}$ takes on average a third of the time of S-phase, giving respective estimates for $a_{2}$ and $a_{3}$. For healthy cells, we take it that there is not an extensive variation in the cell doubling time once they are cycling at maximal rates, and hence $a_{*}^{s}$ is treated deterministically. To estimate the order of magnitude of $T_{s}$ we note that human livers have a typical regeneration time of approximately 3-6 months (Court et al., 2002) but the primary source of toxicity is the heptobilary epithelia, rather than systemic liver failure. This suggests that the toxicity limiting cells have a doubling time that is substantially less than 100-200 days. However, in systemic therapy, the fastest cycling tissues, such as the gut epithelia, are the most sensitive and toxicity limiting (Inomata et al., 2002); these have a doubling time as fast as approximately 1 day (Rew and Wilson, 2000). This indicates that we take $T_{s} \sim 10$ days as an intermediate between these extremes; in the simulations, we specifically work with $T_{s}=35 / 3$ days.

We now consider $\zeta$, the apoptotic rate of quiescent cells. A healthy liver has a mitotic rate of $1: 2 \times 10^{4}$ cells undergoing mitosis at any particular time (Palmes and Spiegel, 2004). This gives a non-cycling death rate of $5 \times 10^{-5}$ per cell cycle assuming that the healthy liver undergoes mitosis solely to replace cells lost by apoptosis. This does not include indirect toxic effects, which would more than likely cause an increase in the non-cycling death rate. We therefore increase this approximation and take $\zeta=2.75 \times 10^{-3}$ per day. The effects on regrowth will still be minimal with this change since $\zeta T_{S} \ll 1$.

Experimentally, it has been observed that, following partial resection, when regrowth occurs, the first surge of DNA replication reaches a maximum approximately $24 \mathrm{~h}$ after initiation (Palmes and Spiegel, 2004). We can therefore assume that the rate of transfer from $\mathrm{G}_{0}$ to $\mathrm{G}_{1}$, denoted $\xi$, would be (at most) $\xi^{-1} \sim 24 \mathrm{~h}$, which gives an order of magnitude estimate for this parameter.

Since in their natural state, the majority of liver cells are quiescent, we take it that $\mu / \xi \gg 1$ so that the rate of becoming quiescent is significantly faster than the rate of becoming active once quiescent. In the simulations, we use $\mu / \xi=27 / 4$ though as long as we have an appropriate order of magnitude for the choice of $\mu$ this choice does not impact the observed trends nor the conclusions.

When cell cycle arrest is terminated, as is the case when reactivation of quiescent cells occurs during regeneration, a period of approximately $10 \mathrm{~h}$ is observed in mammalian cells in culture (Zetterberg et al., 
TABLE A3 A set of reference parameter values for the healthy cell model and the tumour cell model. The parameters $N_{s}$ and $v_{*}$ are further discussed in the text, together with details of the above parameter estimates

\begin{tabular}{|c|c|c|}
\hline Parameter & Value Estimate & Interpretation \\
\hline$\overline{T_{\mathrm{c}} /[1 \mathrm{day}]}$ & $\in\{2.0,3.0,3.5,4.0\}$ & Median tumour cell cycle time. \\
\hline$T_{s}$ & {$[35 / 3]$ days } & Healthy cell cycle time. \\
\hline$v_{*}$ & - & Cell volume immediately after mitosis. \\
\hline$N_{0}$ & $9.9 \times 10^{5}$ & Initial number of proliferating cells in a micrometastasis. \\
\hline$N_{s}$ & - & Initial healthy cell number. \\
\hline$V_{\max }$ & $2 N_{S} v_{*}$ & Maximal volume in the volume regulation mechanism. \\
\hline$a_{*}^{c}$ & $T_{\mathrm{c}}-a_{2}-a_{3}$ & Median $G_{1}$ transit time for the tumour population. \\
\hline$a_{*}^{s}$ & $T_{s}-a_{2}-a_{3}$ & $\begin{array}{l}\text { The } G_{1} \text { transit time under optimal growth conditions for } \\
\text { the healthy cell population. }\end{array}$ \\
\hline$a_{1}$ & $1.5 T_{\mathrm{c}}$ & Maximum duration of the tumour cell $\mathrm{G}_{1}$-phase. \\
\hline$a_{2}$ & $12 \mathrm{~h}$ & S-phase duration. \\
\hline$a_{3}$ & $a_{2} / 3$ & $\mathrm{G}_{2} / \mathrm{M}$ transit time \\
\hline$\alpha$ & $\begin{array}{l}\alpha a_{2} \in\left[10^{-4} \ln (2)\right. \\
\left.10^{-8} \ln (2)\right]\end{array}$ & $\begin{array}{l}\text { Mutation rate of tumour cells in S-phase; estimates vary } \\
\text { widely and different values are considered in the } \\
\text { simulations. }\end{array}$ \\
\hline$\sigma / T_{\mathrm{c}}$ & 0.25 or $1 / \sqrt{8}$ & $\begin{array}{l}\text { A measure of the coefficient of variation for the tumour } \\
\text { cell cycle time. }\end{array}$ \\
\hline$\zeta$ & $0.00275 / 1$ day & The apoptotic rate of healthy cells. \\
\hline$\xi$ & $1 /[1$ day $]$ & The rate of transfer from $G_{0}$ to $G_{1}$ for healthy cells. \\
\hline$\mu$ & $27 \xi / 4$ & The rate of transfer from $G_{1}$ to $G_{0}$ for healthy cells. \\
\hline$\Delta$ & 10 hours & $\begin{array}{l}\text { Time delay between signal and effect for recruitment of } \\
\text { healthy cells into cycle. }\end{array}$ \\
\hline$\chi$ & $0.5 \ln (10) / 1$ day & Chemotherapy induced apoptosis rate. \\
\hline$\Upsilon$ & 0.05 & Toxicity constraint parameter. \\
\hline$t_{1}, k t_{1}$ & Variable & $\begin{array}{l}\text { Respectively, the duration of a chemotherapeutic } \\
\text { administration and the rest phase between } \\
\text { chemotherapeutic administrations. }\end{array}$ \\
\hline$\hat{\psi}, \hat{m}$ & Variable & $\hat{\psi}$ is the cell kill area under curve of a single dose $\hat{\psi}=\hat{m} t_{1}$. \\
\hline$\psi, m$ & Variable & $\begin{array}{l}\psi \text { is the cell kill area under curve of a single, maximally } \\
\text { tolerated, dose, } m \text {; hence } \psi=m t_{1} \text {. }\end{array}$ \\
\hline
\end{tabular}

1995; Wolfe, 1995) which we thus take as an estimate of the time delay for reactivation, $\Delta$. All the above cell cycle parameter estimates are summarized in Table A3.

A.2.2. The cancerous cell population model. Since the data used to obtain estimates of $a_{2}$ and $a_{3}$ for the healthy cell population are independent of population type, we use the same estimates for the tumour population. We have the flexibility to alter the duration of the mean tumour cell cycle time, $T_{\mathrm{c}}$, and consider 


$$
T_{\mathrm{c}} /[1 \text { day }] \in\{2.0,3.0,3.5,4.0\},
$$

the latter two of which are particularly representative of values reported for colorectal cancer (Rew and Wilson, 2000). Once these parameters are specified, the median $\mathrm{G}_{1}$ transit time for the cancer cell, $a_{*}^{c}$, is fixed.

The coefficient of variation for the cell cycle time has been estimated to be between 0.18 and 0.25 for two-cultured tumour cell lines (Chiorino et al., 2001), though one might expect larger variation in situ due to tumour heterogeneity. Noting that the $\mathrm{G}_{1}$ transit time and the tumour cell cycle time differ by a fixed constant, the coefficient of variation for the tumour cell cycle time is approximately $\sigma / T_{\mathrm{c}}$, by inspection of (16). This is not exact only because the Gaussian in (16) is truncated. Given the (under)estimates from the empirical data, we consider $\sigma / T_{\mathrm{c}} \in\{1 / 4,1 / \sqrt{8}\}$ which fixes $\sigma$ and yields coefficients of variation of 0.24 and 0.31 .

The parameter $a_{1}$ is the maximum duration allowed for the $\mathrm{G}_{1}$ phase. Choosing $a_{1}=1.5 T_{\mathrm{c}}$ is a balance between keeping $a_{1}$ sufficiently small to allow tractable computation and allowing a sufficient range for the transit time so as to capture the variation in tumour cell cycle time. With this choice, the maximum $\mathrm{G}_{1}$ transit time is always at least 1.6 standard deviations above the median, so that only a minority $(\sim 3 \%)$ of cells are forced to leave $\mathrm{G}_{1}$ at age $a_{1}$ rather than ageing further in the compartment. A minority of cells also leave the $\mathrm{G}_{1}$ phase earlier than the typical shortest transit time of $6 \mathrm{~h}$ (Abdreeff et al., 2000): in particular, for mean cycle times, $T_{\mathrm{c}}$, of three days or more, at most only $0.2 \%$ of cells leave $\mathrm{G}_{1}$ before 6 hours. In the more extreme case of a median cell cycle time of $T_{\mathrm{c}}=2$ days, this percentage rises to either $3 \%$ when the cell cycle time standard deviation is given by $\sigma / T_{\mathrm{c}}=1 / 2 \sqrt{2}$ and $10 \%$ when $\sigma / T_{\mathrm{c}}=1 / 4$. Note that no conclusions are drawn solely from the latter case, where the use of a truncated Gaussian is clearly a very crude approximation.

The initial total number of sensitive tumour cells, $N_{0}$, is taken to be $9.9 \times 10^{5}$, which is an estimate for the number of proliferating cells present in a spheroid micrometastasis just after vascularization (Gaffney, 2004). Note once more that if the initial conditions are taken to scale with $N_{0}$, the linearity of the tumour cell model ensures that non-dimensionalized model does not depend on $N_{0}$.

The mutation rate per cell cycle is observed to be in the range $\mathrm{O}\left(10^{-7}-10^{-4}\right)$ (Goldie and Coldman, 1998). We often take the dimensional mutation rate per unit time within S-phase, $\alpha$, to be given by $\alpha=10^{-6} \ln (2) / a_{2}$ with the factor of $1 / a_{2}$ normalizing for the assumption that mutation only occurs in S-phase in the model; however, given the multiple orders of magnitude in the possible range of the mutation rate we also vary $\alpha$ markedly in the simulations.

A.2.3. Toxicity and chemotherapy. The protocol rest phase and drug administration time are varied in the simulation as part of an exploration of the influence of scheduling differences. Once these protocol parameters are fixed, the maximally tolerated dose is calculated from the toxicity constraint; this fixes the maximal cell kill area under curve per administration, $\psi$, and hence the maximal cell kill intensity of the dose, $m$. As detailed in Section (2.3.1), the parameter $\Upsilon$, governing the toxicity constraint, is dictated by requiring that the maximally tolerated dose eliminates most of the $\mathrm{G}_{0}$ reserve.

The apoptotic rate, $\chi$, during intravenous infusion of 5-FU varies according to location in murine intestinal crypts, though it decays from a maximum apoptotic index to approximately $10 \%$ of this maximum over of 1-2 days (Inomata et al., 2002). While noting caution over the use of murine data, such observations indicate that the appropriate order of magnitude for the apoptotic rate is

$$
\chi=0.5 \frac{\ln (10)}{1 \text { day }} \text {. }
$$

